\title{
A Generalised Spherical Harmonic Deconvolution to Obtain Cubic Texture from Ultrasonic Wave Speed
}

\author{
Bo Lan ${ }^{1}$, Michael J.S. Lowe ${ }^{2}$, and Fionn P.E. Dunne ${ }^{2}$ \\ ${ }^{1}$ Department of Engineering Science, University of Oxford, UK \\ ${ }^{2}$ Imperial College London, UK
}

\begin{abstract}
In this paper, the spherical harmonic convolution approach for HCP materials [1] is extended into a generalised form for the principal purpose of bulk texture determination in cubic polycrystals from ultrasonic wave speed measurements. It is demonstrated that the wave speed function of a general single crystal convolves with the polycrystal Orientation Distribution Function (ODF) to make the resultant polycrystal wave speed function such that when the three functions are expressed in harmonic expansions, the coefficients of any one function may be determined from the coefficients of the other two. All three Euler angles are taken in account in the description of the ODF such that the theorem applies for all general crystal systems.

The forward problem of predicting polycrystal wave speed with knowledge of single crystal properties and the ODF is solved for all general cases, with validation carried out on cubic textures showing strong sensitivity to texture and excellent quantitative accuracy in predicted wave speed amplitudes. Importantly, it is also revealed by the theorem that the cubic structure is one of only two crystal systems (the other being HCP) whose orientation distributions can be inversely determined from polycrystal wave velocities by virtue of their respective crystal symmetries. Proof of principle is then established by recovering the ODFs of representative cubic textures solely from the wave velocities generated from a computational model using these texture inputs, and excellent accuracies are achieved in the recovered ODF coefficients as well as the resultant pole figures. Hence the methodology is argued to provide a powerful technique for wave propagation studies and bulk texture measurement in cubic polycrystals and beyond.
\end{abstract}

Keywords: texture, generalised spherical convolution, ultrasound, cubic polycrystals. 


\section{Introduction}

Many commonly used artificial or natural materials are polycrystalline, i.e. they consist of aggregates of crystals [2]. According to the symmetries of their unit cells (the elementary and irreducible unit whose periodic repetition forms the lattice of a crystal), crystals can be assigned to seven systems, of which the cubic system is one of the most common and important [3]. The metals $\mathrm{Fe}, \mathrm{Cr}, \mathrm{Al}, \mathrm{Ni}$, for example, all have cubic crystal structures, with the difference among them being that the former two have the Body-Centred Cubic (BCC) structure and the latter two are Face-Centred Cubic (FCC) [4]. At the single crystal level, elastic, plastic, thermal and many more properties of cubic crystals are anisotropic, although sometimes only weakly so [2]. In addition, due to the solidification and deformation processing of cubic materials, almost all resulting polycrystalline aggregates have non-random or preferred orientation distributions [2], with the only possible exception of samples obtained through the powder metallurgy technique. Hence preferred crystallographic orientation distribution, also referred to as texture, becomes one of the major causes of anisotropy in cubic polycrystals [5] and therefore the interest of many researchers [5-7].

Orientation Distribution Functions (ODF), pole figures and inverse pole figures are among the most commonly used methods to describe the texture of a cubic polycrystal, with only the former containing the full texture information, and the combined plots of at least two poles for the latter two are needed for a full representation [8]. Experimental examination techniques of the texture information include X-ray [9] and neutron diffraction and Electron Back Scatter Diffraction (EBSD) [10,11]. However, they all suffer from drawbacks such as being destructive to the samples, only able to measure surface or subsurface textures, expensive and time consuming to conduct, etc. Ultrasonic techniques, which allow bulk and non-destructive tests through polycrystal aggregates by nature [12], are seen as the candidate for the possible development of an alternative method to overcome the aforementioned drawbacks. Extensive research has been conducted in this regard [13-16], but a satisfactory solution to the inverse problem, i.e., the practical methodology to obtain texture information from ultrasonic waves, has remained elusive. The detailed comparison of different texture descriptions and measurement techniques, as well as a brief review of the literature on inverse studies with ultrasonic techniques, can be found in the introduction of our previous paper [1].

In [1], it was found that with a single Hexagonal Close-Packed (HCP) crystal simplified to be elastically isotropic about its c-axis and the ODF of the polycrytal simplified to be the pole distributions of the c-axes, the convolution of the pole distribution and the single crystal velocity function by means of spherical harmonics gave the polycrystal velocity response; this spherical convolution technique enabled the solution for one variable with knowledge of any two of the three variables (c-axes pole distribution, single crystal velocity function, and polycrystal velocity function), hence providing a solution to both the forward and inverse studies for (simplified) HCP polycrystals.

However, the simplification of the ODF to the c-axes pole distributions during the deduction of the theorem for HCP crystals in the previous paper means that it is not applicable to cubic materials, or indeed any more generalised crystal classes, whose orientation presentations need all three Euler angles. This problem is solved in this paper. By taking full account of the three Euler angles, we expand the spherical convolution approach into a generalised form which applies to all crystal classes when performing forward and inverse studies.

The paper is structured in the following order: first, the description of the ODF is given and the single crystal velocity function of a cubic crystal is analyzed; then the analytical model of the relationship between the ODF, single crystal and polycrystal velocity functions is established, followed by the decompositions of the relationship by means of spherical harmonics and Wigner-D functions, which lead 
to the generalised spherical convolution theorem. The approach is then applied in forward studies for both theoretical distributions of cubic crystals and three experimental polycrystal textures. Finally, proof of principle is established for the experimental BCC textures for the solution of the inverse problem; that is, texture, or determination of the orientation distribution function from ultrasonic analyses.

\section{Background}

\subsection{Orientation distribution function}

The specific orientation of a crystal within a polycrystalline sample is fully captured by means of the three Euler angles, as shown in Figure 1, where there are two sets of coordinate systems defined: one is the sample system $o-x y z$, whose $x, y$ and $z$ axes are often taken to be along the rolling (RD), transverse (TD) and normal (ND) directions of the sample respectively; and the other is the crystal system $o-X Y Z$ associated with the crystal with an arbitrarily rotated orientation from the sample system. The Euler angles $\beta, \alpha$ and $\gamma$ are then employed to apply the rotation from $o-x y z$ to $o-X Y Z$ following the sequences $Z-Y^{\prime}-Z^{\prime \prime}$ of the rotation axes used in [8] and [17].

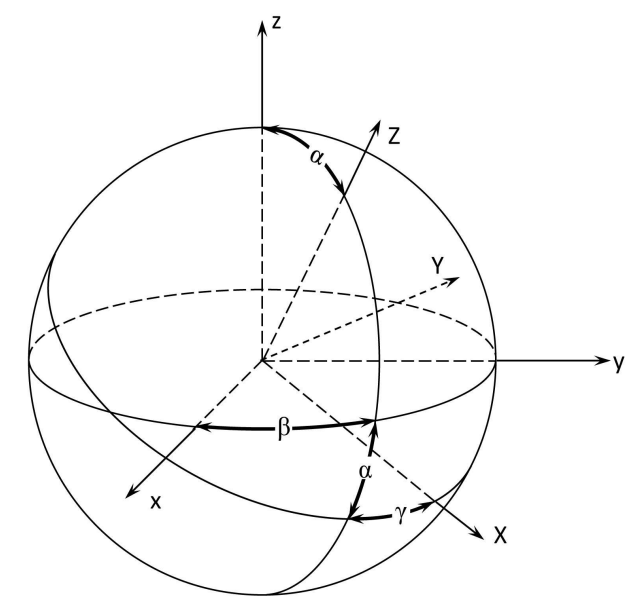

Figure 1: The three Euler angles to rotate the sample system o- $x y z$ to the crystal system $O-X Y Z$.

Therefore, the statistical distribution of the crystallographic orientations within the sample can be described by a probability function of the Euler angles. Often referred to as the orientation distribution function $(\mathrm{ODF})$, the function can be written as $w(\alpha, \beta, \gamma)$ satisfying the following requirement:

$$
\int_{0}^{2 \pi} \int_{0}^{2 \pi} \int_{0}^{\pi} w(\alpha, \beta, \gamma) \sin \alpha d \alpha d \beta d \gamma=1
$$

\subsection{Velocities of ultrasonic waves in a single crystal}

In order to investigate the ultrasonic wave velocity propagating through a single crystal along an arbitrary direction in three-dimensional space, the elastic stiffnesses of the crystal are needed. A single crystal $\alpha$-phased Fe, for example, has the following stiffness matrix(with units of GPa) at room 
temperature [4]:

$$
C=\left(\begin{array}{llllll}
228.09 & 133.48 & 133.48 & & & \\
133.48 & 228.09 & 133.48 & & & \\
133.48 & 133.48 & 228.09 & & & \\
& & & 110.87 & & \\
& & & & 110.87 & \\
& & & & & 110.87
\end{array}\right)
$$

The ultrasonic wave velocity in any given direction $\mathbf{n}=\left(\begin{array}{lll}\mathbf{n}_{\mathbf{1}} & \mathbf{n}_{\mathbf{2}} & \mathbf{n}_{\mathbf{3}}\end{array}\right)^{\mathbf{T}}$ can then be calculated through the well-known Christoffel equation by substituting 2 and $\mathbf{n}$ into the equations given in the appendices of [18], which ultimately leads to a classic eigenvalue-eigenvector problem, with the eigenvalues of the Christoffel matrix directly linked to three phase velocities (one longitudinal and the other two shear) and the three eigenvectors being the respective particle displacement directions corresponding to the phase velocities.

Note that our principal interests in this study lie with the fastest bulk wave; that is, the longitudinal wave. However, in an arbitrary direction $\mathbf{n}$ within generally anisotropic materials, even though the three particle displacement vectors are mutually orthogonal, none of them actually lies parallel with $\mathbf{n}$, meaning that the fastest phase velocity obtained through the Christoffel equation is in fact quasi-longitudinal. It is shown in the Appendix that while the angular difference between $\mathbf{n}$ and its closest eigenvector is generally small and negligible for HCP materials simplified to be transversely isotropic, it can be rather significant for cubic materials whose anisotropies are more pronounced. It is important to be aware of this difference in the studies of this paper, and this will be discussed further in Section 4.1.2.
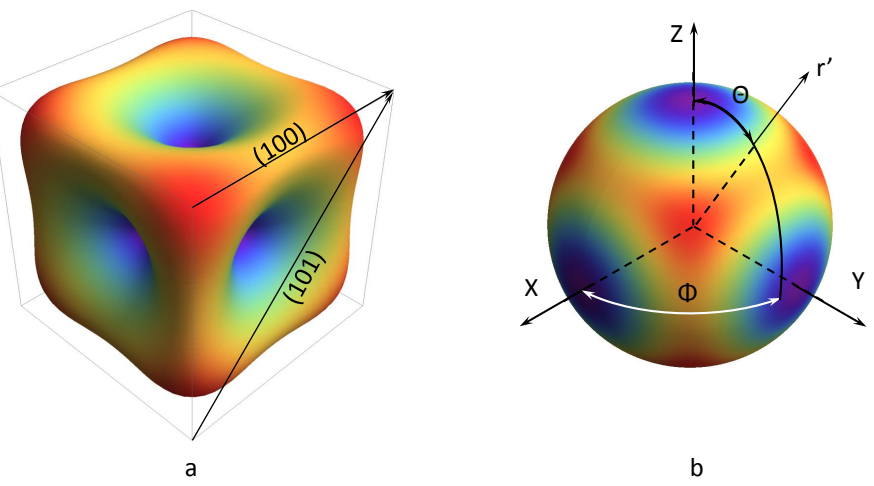

Figure 2: The single crystal velocity surface of Fe, with: a. the velocity variation as the explicit surface shape; b. as the colour change. The single crystal velocity in an arbitrary direction $\boldsymbol{r}^{\prime}$ is determined by its polar angle $\Theta$ and azimuthal angle $\Phi$ with respect to the crystal system $o-X Y Z$.

The ultrasonic wave velocities of a single crystal in all directions of the space form its velocity surface, which is also a rough evaluation of the elastic stiffnesses-to-density ratio of the crystal given the dependence of the wave velocity on the ratio. The single crystal velocity surface of $\alpha$-phased Fe is plotted in Figure 2, with the velocity variations shown as explicit shape change of the surface in 
Figure 2a and colour variations on the surface of the unit sphere in Figure 2b. It can be seen that all the symmetries of a cubic crystal, including four-fold symmetries about the $Z$-axis and all the mirror planes, are well preserved on the velocity surface, which agrees with Neumann's principle [19]. Moreover, the wave velocity in an arbitrary direction $\boldsymbol{r}^{\prime}$ is determined by the combination of its polar angle $\Theta$ and azimuthal angle $\Phi$ with respect to the crystal system $o-X Y Z$. So if the single crystal velocity surface is written as a function $k$, the velocity in direction $\boldsymbol{r}^{\prime}$ is

$$
k\left(\boldsymbol{r}^{\prime}\right)=k(\Theta, \Phi)
$$

\section{Theoretical Framework}

In this section, the analytical model and the relationship between the single crystal wave velocity function, ODF and polycrystal velocity function for general crystal systems are constructed. Similar ideas to those presented in [1] are employed, but now with full consideration of all three Euler angles.

\subsection{Refinement of the grain average model}

When an ultrasonic wave is excited into a sample from a point source, its three-dimensional wavefront expands out approximately as a sphere, with some distortions introduced by the anisotropy within the polycrystal; and if only the wave propagation in a certain direction is of interest, the problem can be simplified to be a wave going through an array of crystals in a straight line [1].

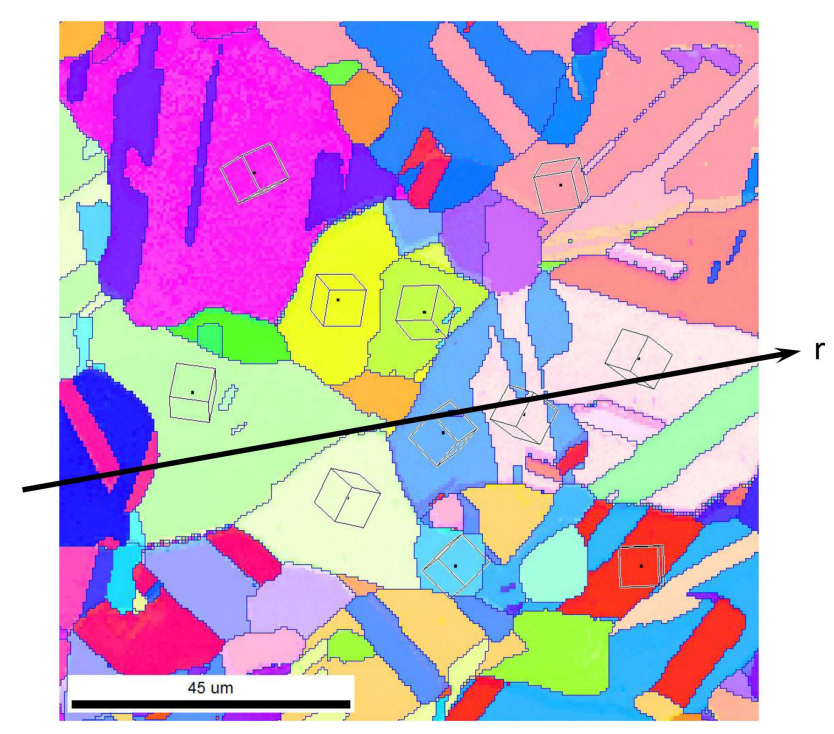

Figure 3: A wave going through a polycrystalline aggregate in direction $\boldsymbol{r}$. The boxes on the crystals represents the respective crystallographic orientations (EBSD grain map courtesy of Dr Jun Jiang, Dr Ben Britton and Professor Angus Wilkinson [20]).

Consider the case shown in Figure 3 where the wave goes through a cubic polycrystalline aggregate in the direction of $\boldsymbol{r}$, with the mini-cubes on each crystal in the figure representing its crystallographic orientation. The wave velocities in different crystals are different, and the overall velocity through the aggregate can be calculated via the grain average method. Each crystal introduces a local coordinate system $o-X_{i} Y_{i} Z_{i}$, about which the wave propagation direction $\boldsymbol{r}$ is evaluated to give the velocity 
through this particular crystal; then the contributions to the overall velocity in the direction from all grains are summed up one by one with regards to the volume fractions of their specific crystallographic orientations. The wave velocity in the $i$ th crystal is determined in such a way that if the direction $\boldsymbol{r}$ with respect to the local crystal system is denoted as $\boldsymbol{r}_{\boldsymbol{i}}^{\prime}$, and the latter is described by its polar angle $\Theta_{i}$ and azimuthal angle $\Phi_{i}$, then the wave velocity is $k\left(\Theta_{i}, \Phi_{i}\right)$ according to equation 3. Meanwhile, if the volume fraction of crystals with the orientation $(\alpha, \beta, \gamma)$ is described by a function $g(\alpha, \beta, \gamma)$, the overall wave velocity through the aggregate in $\boldsymbol{r}$ can be calculated by:

$$
v(\boldsymbol{r})=\sum k\left(\boldsymbol{r}_{\boldsymbol{i}}^{\prime}\right) \cdot g\left(\alpha_{i}, \beta_{i}, \gamma_{i}\right)
$$

In reality, however, the crystallographic orientation does not necessarily remain uniform even within the same grain, so here we allow the orientations to vary continuously rather than keeping the averaging unit as a whole grain. As a result, the summation in equation 4 becomes an integration:

$$
v(\boldsymbol{r})=\int_{0}^{2 \pi} \int_{0}^{2 \pi} \int_{0}^{\pi} k\left(\boldsymbol{r}^{\prime}\right) \cdot g(\alpha, \beta, \gamma) \sin \alpha d \alpha d \beta d \gamma
$$

Now consider the wave propagations through a full three-dimensional polycrystalline aggregate which is statistically homogeneous in such a way that when evaluating from any direction in the space, the waves all go through the same texture. The analytical equation 5 also applies for this case if we allow $\boldsymbol{r}$ to cover all the directions in the space.

The $\boldsymbol{r}$ on the left-hand side of equation 5 is the wave incident direction described by its polar angle $\theta$ and azimuthal angle $\phi$ with respect to the sample system $o-x y z$; the $\boldsymbol{r}^{\prime}$ on the right-hand side of the equation is essentially the same direction vector as $\boldsymbol{r}$, but given with respect to the crystal system $o-X Y Z$ and is described by $\Theta$ and $\Phi$. In addition, $o-X Y Z$ is linked with $o-x y z$ via the rotation of the coordinate system using the Euler angles $(\alpha, \beta, \gamma)$, which, according to the conventions in quantum mechanics [21], is a passive rotation, and its corresponding active operator $R^{(\alpha, \beta, \gamma)}$ to rotate the wave incident direction vector $\boldsymbol{r}$ to $\boldsymbol{r}^{\prime}$ can be constructed accordingly using the same set of Euler angles:

$$
(\Theta, \Phi)=R^{(a)}(\alpha, \beta, \gamma) \cdot(\theta, \phi)=R_{z}^{(a)}(\beta) \cdot R_{y}^{(a)}(\alpha) \cdot R_{z}^{(a)}(\gamma) \cdot(\theta, \phi)
$$

where $R_{z}^{-\beta}$, for example, means to rotate the vector about the z-axis with an offset of $-\beta$.

Now that the orientation $(\alpha, \beta, \gamma)$ varies continuously in the three-dimensional space, the volume fraction function $g(\alpha, \beta, \gamma)$ follows the same concept with the ODF $w$ defined in equation 1. Replacing $g$ with $w$ in equation 5 and substituting equation 6 back into 5 leads to:

$$
\begin{aligned}
v(\theta, \phi) & =\int_{0}^{2 \pi} \int_{0}^{2 \pi} \int_{0}^{\pi} k(\Theta, \Phi) w(\alpha, \beta, \gamma) \sin \alpha d \alpha d \beta d \gamma \\
& =\int_{0}^{2 \pi} \int_{0}^{2 \pi} \int_{0}^{\pi} k\left(R^{(a)}(\alpha, \beta, \gamma) \cdot(\theta, \phi)\right) w(\alpha, \beta, \gamma) \sin \alpha d \alpha d \beta d \gamma
\end{aligned}
$$

This is the final grain average analytical model to describe the relationship between polycrystal velocity function $v$, the single crystal velocity function $k$ and the ODF $w$ in the three-dimensional space. The function $k$ serves as a bridge to connect $v$ and $w$ and is therefore called the kernel of the relationship. 


\subsection{Generalised spherical convolution theorem}

The relationship shown in equation 7 can be further exploited by means of the spherical harmonic (SH) expansion. As the analogues on the surface of a sphere to the Fourier bases on a line or a circle, the spherical harmonics are defined as:

$$
Y_{l m}(\theta, \phi)=P_{l m}(\cos \theta) e^{i m \phi}
$$

where $l(l \geq=0)$ and $m(-l \leq m \leq l)$ are called the degree and order of the basis. $P_{l m}(\cos (\theta))$ is the normalized associate Legendre function accounting for the effects of the polar angle $\theta$, and $e^{i m \phi}$ means that the dependence on the azimuthal angle $\phi$ is expanded as the Fourier bases in the spherical harmonics. Note that the Condon-Shortley phase is excluded from the spherical harmonics used in this paper. We now begin the analysis by expanding the kernel $k(\Theta, \Phi)$ in equation 7 in terms of the spherical harmonics associated with the local crystal system o- $X Y Z$ :

$$
k(\Theta, \Phi)=\sum_{l=0}^{\infty} \sum_{n=-l}^{l} K_{l n} Y_{l n}(\Theta, \Phi)
$$

where the $\mathrm{SH}$ coefficients $K_{l n}$ are calculated by projecting the original function onto the complex conjugates of the bases:

$$
K_{l n}=\int_{0}^{2 \pi} \int_{0}^{\pi} k(\Theta, \Phi) Y_{l n}^{*}(\Theta, \Phi) \sin \Theta d \Theta d \Phi
$$

where $Y_{l n}^{*}(\Theta, \Phi)$ stands for the complex conjugate of $Y_{l n}(\Theta, \Phi)$.

The next step is to establish connections between the expansion in equation 9 , which is with respect to the crystal system, and the sample system $o-x y z$. It can be done with the help of the Wigner-D matrix $D^{l}(\alpha, \beta, \gamma)$, which is a $(2 l+1) \times(2 l+1)$ dimensioned matrix containing the information to express a set of $l$-degree spherical harmonics as the linear combinations of another set of rotated l-degree harmonics. The elements of the Wigner-D matrix are defined as:

$$
D_{m n}^{l}(\alpha, \beta, \gamma)=e^{i m \beta} d_{m n}^{l}(\alpha) e^{i n \gamma}
$$

where $-l \leq m, n \leq l$, and the $d_{m n}^{l}(\alpha)$ are the elements of the Wigner-d matrix directly related to the Jacobi polynomials. $d_{m n}^{l}(\alpha)$ follow a similar concept with $Z_{l m n}(\cos \alpha)$ defined by Roe in [8], but are not normalized. Now $Y_{l n}(\Theta, \Phi)$ can be related to the harmonics with respect to the sample system through the Wigner-D matrices, as shown in [22]:

$$
Y_{l n}(\Theta, \Phi)=\sum_{m=-l}^{l} D_{m n}^{l *}(\alpha, \beta, \gamma) Y_{l m}(\theta, \phi)
$$

Substituting equation 12 back to 9 makes the kernel function $k(\theta, \phi)$ further expanded into

$$
k(\Theta, \Phi)=\sum_{l=0}^{\infty} \sum_{n=-l}^{l} K_{l n} \sum_{m=-l}^{l} D_{m n}^{l *}(\alpha, \beta, \gamma) Y_{l m}(\theta, \phi)
$$

Now we turn our focus to the $\operatorname{ODF} w(\alpha, \beta, \gamma)$. As shown by Kostelec et al [23], the Wigner-D matrices also form a complete set of orthogonal bases, and the ODF, or indeed any function of the rotation 
group $\mathrm{SO}(3)$ defined on the three Euler angles, have the following decomposition:

$$
w(\alpha, \beta, \gamma)=\sum_{l=0}^{\infty} \sum_{m=-l}^{l} \sum_{n=-l}^{l} \frac{2 l+1}{8 \pi^{2}} \cdot W_{l m n} D_{m n}^{l}(\alpha, \beta, \gamma)
$$

where the coefficients $\frac{2 l+1}{8 \pi^{2}}$ result from the fact that the Wigner-D matrices are not normalized, and the expansion coefficients $W_{l m n}$ are obtained via:

$$
W_{l m n}=\int_{0}^{2 \pi} \int_{0}^{2 \pi} \int_{0}^{\pi} w(\alpha, \beta, \gamma) D_{m n}^{l *}(\alpha, \beta, \gamma) \sin \alpha d \alpha d \beta d \gamma
$$

Here $D_{m n}^{l *}(\alpha, \beta, \gamma)$ stands for the complex conjugate of $D_{m n}^{l}(\alpha, \beta, \gamma)$.

Substituting equations 13 and 14 back into 7 transforms the latter into:

$$
\begin{aligned}
& v(\theta, \phi)=\int_{0}^{2 \pi} \int_{0}^{2 \pi} \int_{0}^{\pi} k\left(R^{(a)}(\alpha, \beta, \gamma) \cdot(\theta, \phi)\right) w(\alpha, \beta, \gamma) \sin \alpha d \alpha d \beta d \gamma \\
& =\sum_{l_{1}=0}^{\infty} \sum_{m=-l_{1}}^{l_{1}} \sum_{n_{1}=-l_{1}}^{l_{1}} K_{l_{1} n_{1}} Y_{l_{1} m_{1}}(\theta, \phi) \cdot \sum_{l_{2}=0}^{\infty} \sum_{m_{2}=-l_{2}}^{l_{2}} \sum_{n_{2}=-l_{2}}^{l_{2}} \frac{2 l+1}{8 \pi^{2}} W_{l_{2} m_{2} n_{2}} \cdot T_{l_{1}, n_{1}, n_{1}, l_{2}, m_{2}, n_{2}}
\end{aligned}
$$

and the term $T_{l_{1}, n_{1}, n_{1}, l_{2}, m_{2}, n_{2}}$ can be simplified according to the orthogonality (but not normalization) of the Wigner-D matrix into:

$$
\begin{aligned}
T_{l_{1}, n_{1}, n_{1}, l_{2}, m_{2}, n_{2}} & =\int_{0}^{2 \pi} \int_{0}^{2 \pi} \int_{0}^{\pi} D_{m_{1} n_{1}}^{l_{1} *}(\alpha, \beta, \gamma) \cdot D_{m_{2} n_{2}}^{l_{2}}(\alpha, \beta, \gamma) \sin \alpha d \alpha d \beta d \gamma \\
& =\frac{8 \pi^{2}}{2 l+1} \delta_{l_{1}, l_{2}} \delta_{m_{1}, m_{2}} \delta_{n_{1}, n_{2}}
\end{aligned}
$$

where $\delta_{a, b}$ is the Kronecker delta function equal to 1 if and only if $a=b$. So equation 17 enforces $l_{1}=l_{2}, m_{1}=m_{2}$ and $n_{1}=n_{2}$, which turns equation 16 into:

$$
v(\theta, \phi)=\sum_{l=0}^{\infty} \sum_{m=-l}^{l} \sum_{n=-l}^{l} W_{l m n} K_{l n} \cdot Y_{l m}(\theta, \phi)
$$

And this means that if the polycrsytal velocity function $v$ is decomposed in terms of spherical harmonics, its coefficients are:

$$
V_{l m}=\sum_{n=-l}^{l} W_{l m n} K_{l n}
$$

Equations 18 and 19 form the generalised convolution theorem for the analysis of the relationship between the polycrystal velocities, single crystal velocities and the crystallographic orientation distribution function.

\subsection{Discussions of the approach}

\subsubsection{Normalization conventions of $W_{l m n}$}

There are different normalization conventions for the decomposition bases and $W_{l m n}$ in the literature. Roe [8], for example, used normalized Wigner-D matrices as the decomposition bases, therefore the 
coefficients obtained in [8] have the following relationship with the $W_{l m n}$ used in this paper:

$$
W_{l m n}=\frac{2}{2 l+1} \cdot W_{l m n}(\text { Roe's })
$$

The most widely used normalization convention of $W_{l m n}$ in the metal industries, however, is half of Roe's such that the ODF $w(\alpha, \beta, \gamma)$ always exhibit unity when the texture is random [24]. The relationship between this convention and our $W_{l m n}$ is therefore:

$$
W_{l m n}=\frac{1}{2 l+1} \cdot W_{l m n}(\text { Industrial })
$$

\subsubsection{Using the theorem for forward studies}

The deductions of equations 18 and 19 have taken full account of all three Euler angles, which, no matter what type of structure a crystal belongs to, provides enough information to describe its exact orientation. Hence the theorem applies to all types of crystal structures.

When the texture information is known, applying equation 19 from the right side to the left is the forward study process: the ODF expansion coefficients $W_{l m n}$ can be calculated from the texture information using equation 15 (for which the MTEX toolbox for Matlab [25] is a very useful tool); then the SH coefficients $V_{l m}$ of the polycrystal velocity function are obtained by inputting the coefficients of the ODF and the kernel (single crystal velocity function) to equation 19; finally, the polycrystal velocity function is reconstructed based on the obtained $V_{l m}$, the velocity surface is established and the wave speeds in all directions of the space are predicted. Again, this process can be done for all types of crystal structures.

However, for the inverse study process where the polycrystal velocity function is known and the ODF is needed, it is not possible for all types of crystal structures to retrieve the ODF coefficients by using the theorem from the left side to the right. The reason is that for an arbitrary crystal structure, there are $2 l+1$ unknown variables $W_{l m n}(-l \leq n \leq l)$ for a given set of $l$ and $m$ to be solved from equation 19 only, which is not theoretically possible. However, there are two exceptions, being HCP and cubic crystal structures, whose ODF coefficients $W_{l m n}$ both have only one independent unknown variable (at least up to a certain $l$ ) for a given set of $l$ and $m$, but in two different ways. Therefore equation 19 can be solved and the ODF information can be recovered from the polycrystal velocity functions for these two types of crystals.

\subsubsection{Using the theorem for inverse studies on HCP materials}

The inverse study on HCP materials was fully analysed in our previous paper [1], and it can be proved that the spherical convolution theorem obtained specifically for HCP materials is actually a special case of the generalised convolution theorem described by equations 18 and 19 .

As an HCP crystal is often simplified as transversely isotropic about its c-axis, only one angle in the crystal system is needed to determine the wave velocity in a given direction; that is, the polar angle $\Theta$ (which is also the angle between the wave direction and the c-axis of the crystal), and the kernel has no dependence on the azimuthal angle $\Phi$. This means the coefficients of the kernel $K_{l n}$ have non-zero values only when $n=0$, which simplifies equation 19 to be:

$$
V_{l m}=W_{l m 0} K_{l 0}
$$


In addition, if the distribution intensity of the (0001) pole within an HCP aggregate is denoted as a function $q$, then its $\mathrm{SH}$ coefficients $Q_{l m}$ are related with the ODF coefficients $W_{l m 0}$ by:

$$
Q_{l m}=\sqrt{\frac{2 l+1}{4 \pi}} \cdot W_{l m 0}
$$

Substituting equation 23 back into 22 makes the latter into the spherical convolution theorem for HCP materials in [1], which proves the compatibility of the generalised theorem with HCP materials and that the HCP theorem is indeed a special case of the generalised one.

\subsubsection{Using the theorem for inverse studies on cubic materials}

With all the symmetries of a cubic crystal considered, there are in total 24 equivalences for an arbitrary crystallographic orientation, and they must be taken correct account of in order to obtain accurate results of the ODF coefficients for a polycrystal. This topic has been thoroughly studied in Roe's papers [8,26], and some of the most important symmetries as well as their effects on the ODF coefficients are listed in Table 1.

It is shown in Table 1 that the number of unknown non-zero coefficients $W_{l m n}$ has been reduced due to

Table 1: Effects of cubic crystal symmetries on the ODF coefficients, part I $[8,26]$

\begin{tabular}{|c|c|c|}
\hline Crystallographic symmetry elements & \multicolumn{2}{|c|}{ The effects on $W_{l m n}$} \\
\hline $\begin{array}{l}\text { Antipodal symmetry (also referred to } \\
\text { as the centrosymmetry), i.e. }(\Theta, \Phi)= \\
(\Theta+\pi, \Phi+\pi)\end{array}$ & $W_{l m n}=\left\{\begin{array}{l}W_{l m n}, \\
0\end{array}\right.$ & $\begin{array}{l}l \text { even } \\
l \text { odd }\end{array}$ \\
\hline $\begin{array}{l}\text { Mirror planes } \perp X, Y, Z \text {, and four-fold } \\
\text { rotation symmetry about } Z\end{array}$ & $\begin{array}{r}W_{l m n}=\left\{\begin{array}{l}W_{l m \bar{n}}, \\
0,\end{array}\right. \\
\text { where } \mathrm{k} \text { is int }\end{array}$ & $\begin{array}{l}n=4 k \\
n \neq 4 k \\
\text { eger }\end{array}$ \\
\hline
\end{tabular}

symmetries, with $W_{l m n}$ being so only when $l$ is even and $n=4 k$; In addition, the rotation invariance of the crystallographic orientations of a cubic crystal when the Euler angles are $\alpha=0, \beta=\frac{\pi}{2}, \gamma=0$ further enforce linear relationships on the remaining non-zero $W_{l m n}$, as proved by Roe [26] and listed in Table 2. Note that only the relationships for $W_{l m n}$ with $l$ up to 10 are shown here, and the ones for larger $l$ s can be found in Roe's paper [26].

Tables 1 and 2 demonstrate that for a certain set of $l(l \leq 10)$ and $m(-l \leq m \leq l)$, there is only one linearly independent ODF coefficient $W_{l m 0}$, and all the other $W_{l m n}$ are either equal to 0 or linearly dependent on $W_{l m 0}$. In fact, there are more linear dependences for $W_{l m n}$. The relationship $W_{l m \bar{n}}=W_{l m n}$ in Table 2 can be proved to lead to a further implication such that:

$$
W_{l \bar{m} n}=W_{l m n}^{*}
$$

where $\bar{m}$ in $W_{l \bar{m} n}$ means $-m$, and $W_{l m n}^{*}$ refers to the complex conjugate of $W_{l m n}$. So with considerations on all the symmetries, we come to the conclusion that $W_{l m 0}$ with $l \leq 10$ and $0 \leq m \leq l$ contain all the linearly independent terms needed to reconstruct the original ODF matrices. Therefore, theoretically, for these sets of $l$ and $m$, equation 19 can be solved to obtain all the ODF coefficients, and based on the results the ODF band-limited to $l \leq 10$ can then be reconstructed.

A further important point is that the kernel (single crystal velocity) function of a cubic crystal shares 
Table 2: Effects of cubic crystal symmetries on the ODF coefficients, part II [26]

\begin{tabular}{cll}
\hline \hline degree $l$ & $\begin{array}{c}\text { Linearly indepen- } \\
\text { dent coefficients }\end{array}$ & The other coefficients \\
\hline 0 & $W_{000}$ & \\
\hline 2 & None & $W_{2 m n}=0$ \\
\hline 4 & $W_{4 m 0}$ & $W_{4 m 4}=0.59761430 \cdot W_{4 m 0}$ \\
\hline 6 & $W_{6 m 0}$ & $W_{6 m 4}=-1.8708287 \cdot W_{6 m 0}$ \\
\hline 8 & $W_{8 m 0}$ & $W_{8 m 4}=0.37605072 \cdot W_{8 m 0}$ \\
\hline 10 & $W_{8 m 8}=0.57295971 \cdot W_{8 m 0}$ \\
\hline \hline
\end{tabular}

all the symmetries listed in Tables 1 and 2, as shown by Figure 2, so all the effects of the symmetries in these tables are equally applicable to the kernel $\mathrm{SH}$ coefficients. For example, $K_{l n}$ have non-zero value only when $l \neq 0$ and $n=4 k$, and $K_{44}=0.59761430 \cdot K_{40}$, etc.

As an example to demonstrate more explicitly how the generalised spherical convolution theorem works on cubic materials, it is sensible to write equation 19 with $l=4$ and $-4 \leq m \leq 4$ in matrix form as:

$$
\left(\begin{array}{cccc:c:cccc}
W_{4 \overline{4} \overline{4}} & 0 & 0 & 0 & W_{4 \overline{4} 0} & 0 & 0 & 0 & W_{4 \overline{4} 4} \\
W_{4 \overline{3} \overline{4}} & 0 & 0 & 0 & W_{4 \overline{3} 0} & 0 & 0 & 0 & W_{4 \overline{3} 4} \\
W_{4 \overline{2} \overline{4}} & 0 & 0 & 0 & W_{4 \overline{2} 0} & 0 & 0 & 0 & W_{4 \overline{2} 4} \\
W_{4 \overline{1} \overline{4}} & 0 & 0 & 0 & W_{4 \overline{1} 0} & 0 & 0 & 0 & W_{4 \overline{1} 4} \\
W_{40 \overline{4}} & 0 & 0 & 0 & W_{400} & 0 & 0 & 0 & W_{404} \\
W_{41 \overline{4}} & 0 & 0 & 0 & W_{410} & 0 & 0 & 0 & W_{414} \\
W_{42 \overline{4}} & 0 & 0 & 0 & W_{420} & 0 & 0 & 0 & W_{424} \\
W_{43 \overline{4}} & 0 & 0 & 0 & W_{430} & 0 & 0 & 0 & W_{434} \\
W_{44 \overline{4}} & 0 & 0 & 0 & W_{440} & 0 & 0 & 0 & W_{444}
\end{array}\right)\left(\begin{array}{c}
K_{4 \overline{4}} \\
0 \\
0 \\
0 \\
K_{40} \\
0 \\
0 \\
0 \\
K_{44}
\end{array}\right)=\left(\begin{array}{c}
V_{4 \overline{4}} \\
V_{4 \overline{3}} \\
V_{4 \overline{2}} \\
V_{4 \overline{1}} \\
V_{40} \\
V_{41} \\
V_{42} \\
V_{43} \\
V_{44}
\end{array}\right)
$$

In the $W_{4 m n}$ matrix of equation $25, m$ changes with rows and $n$ with columns; while in the kernel vector $K_{l n} \mathrm{n}$ changes with rows. It can be seen that both $W_{4 m n}$ and $K_{l n}$ have non-zeros only when $n=-4,0$ or 4 , while SH coefficients $V_{l m}$ of the polycrystal velocity function can be non-zero for any $m$. Moreover, for the ODF coefficients $W_{4 m n}$, only the ones in the dashed box of equation 25 are linearly independent, so when $V_{l m}$ are known, these equations can be solved one by one to obtain the full $W_{4 \bar{m} n}$ matrix. This is an example with $l=4$, but it works in the same way for all $l \leq 10$.

It is also worth pointing out that only cubic crystal symmetries have been used to aid solving the inverse problem so far, and no statistical sample symmetries have been taken into account. This means the theorem applies to all types of sample symmetries; and if there is any sample symmetry affecting the ODF coefficients, it will manifest itself in the results obtained.

\subsubsection{The flexibility to change the kernel in the theorem}

In [1] it was argued that the spherical convolution approach for HCP materials had the flexibility to change the convolution kernel from ultrasonic phase velocities to slowness, group velocity, or Young's 
modulus, etc, and it is the same for the generalised spherical convolution approach in this paper. So despite that ultrasonic wave phase velocities have been used as the kernel during the deduction of the theorem equation 19, slowness, which is a commonly used concept to evaluate ultrasonic wave phase and group velocities in the non-destructive testing world, can also be used as the kernel to calculate the phase velocity variations in polycrystals; and single crystal group velocity and Young's modulus can be used as the kernel to calculate their respective resulting properties in polycrystals.

In [1] it was also demonstrated that the results obtained by adopting phase velocity and slowness kernels did not in fact display significant differences as far as the longitudinal wave velocities were concerned, and that using wave velocity and slowness as kernels yielded very close representation to the actual case compared to the Voigt method. So in the following sections of this paper, ultrasonic phase velocity is again adopted as the kernel in the theorem and no further comparisons between different methods are performed.

\section{Forward studies: from textures to wave velocities}

As mentioned before, if the ODF of a texture is known, using the generalised theorem in equation 19 from the right side to the left is the forward problem, where the texture information is utilized to predict the polycrystal velocities. In doing so, the kernel, which is the single crystal velocity function, is essential.

As pointed out in Section 2, the velocities of compression waves are proportional to the square root of the elastic stiffnesses-to-density ratio along the wave directions, and therefore the elastic moduli of a single crystal in different directions, which can also form a surface, are able to give us some hints about the actual velocity surface. A comprehensive study on the Young's modulus surfaces of a series of cubic materials, including both body-centred cubic (BCC) and face-centred cubic (FCC) crystals, was conducted by Zhang et al [4], and it was shown that even though the crystal structures of BCC and FCC are different (hence so are the plastic behaviours), the elastic properties of a BCC material can be similar to an FCC one and vice versa. For example, $\alpha$-phased Fe, which is BCC-structured, has a similar single crystal Young's modulus surface (hence also the compression wave speed surface) with a group of FCC materials including $\mathrm{Cu}, \mathrm{Ni}, \mathrm{Au}, \mathrm{Ag}$, etc. On the other hand, the FCC-structured Al has a similar Young's modulus surface compared with $\mathrm{Nb}, \mathrm{Mo}, \mathrm{V}$, which are all BCC; there is also a special material, W, with its single crystal almost being elastically isotropic and its Young's modulus surface approximately spherical.

For the material $\mathrm{W}$, it is easy to conclude that its polycrystal longitudinal wave velocities have no dependence on texture since its single crystal response is almost isotropic. So in this section, our interests are in the other two groups of materials. We start by revealing more information on the single crystal SH coefficients of these materials, and then pick out Fe as the exemplary material on which to perform the forward studies, in the hope of primarily showing how the forward studies are carried out through the generalised convolution theorem, and also revealing some more subtle relationships lying between texture and polycrystal wave speeds.

\subsection{Single crystal velocity surfaces and coefficients}

\subsubsection{Theoretical analysis}

With knowledge of the elastic moduli or the compliance of a single crystal, the velocity surface of the crystal can be determined through the Christoffel equation, and the SH coefficients of the obtained velocity surface are then calculated by expanding the surface in terms of the spherical harmonics 
using equation 10.

Table 3: $\mathrm{SH}$ coefficients for single crystal $\mathrm{Fe}, \mathrm{Cu}$ and $\mathrm{Ni}$

\begin{tabular}{ccccccc}
\hline \hline & $K_{00}$ & $K_{20}$ & $K_{40}$ & $K_{60}$ & $K_{80}$ & $K_{10,0}$ \\
\hline $\mathrm{Fe}$ & 21303.6 & 0.0 & -611.8 & -4.2 & -52.2 & -2.2 \\
$\mathrm{Cu}$ & 17324.1 & 0.0 & -535.5 & -3.9 & -46.5 & -2.0 \\
$\mathrm{Ni}$ & 20877.2 & 0.0 & -642.0 & -4.6 & -59.1 & -2.7 \\
\hline
\end{tabular}

The SH coefficients for the single crystals of the first group of materials, including $\mathrm{Fe}, \mathrm{Cu}$ and $\mathrm{Ni}$, are obtained with this method using the compliance data in [4], and their $K_{l 0}$ for $l \leq 10$ are listed in Table 3. All the other non-zero coefficients of these degrees can also be retrieved though the relationships in Table 1 and 2 .

It is evident in Table 3 that for this group of materials, the 2nd degree SH coefficients are enforced to be 0 by the crystal symmetries, and the amplitudes of the higher degree ones $K_{l n}$ do not decrease monotonically with the degree $l$, but as two separate sets, ie the $4 k$-th and the $(4 k+2)$-th degrees. The 8 th degree terms, for example, have an amplitude much larger than both the 6 th and 10th degree terms.

Table 4: SH coefficients for single crystal Al, $\mathrm{V}$ and $\mathrm{Nb}$

\begin{tabular}{ccccccc}
\hline \hline & $K_{00}$ & $K_{20}$ & $K_{40}$ & $K_{60}$ & $K_{80}$ & $K_{10,0}$ \\
\hline $\mathrm{Al}$ & 23237.70 & 0.00 & -122.24 & -0.18 & -0.63 & -0.01 \\
$\mathrm{~V}$ & 21382.30 & 0.00 & 156.73 & -0.33 & -2.92 & 0.02 \\
$\mathrm{Nb}$ & 18171.10 & 0.00 & 296.11 & -1.42 & -11.65 & 0.26 \\
\hline \hline
\end{tabular}

Similarly, the SH coefficients for the single crystals of the other group of materials, including $\mathrm{Al}, \mathrm{Nb}$ and V, can also be obtained using this method, and their $K_{l 0}$ are listed in Table 4. It can be seen that compared to the first group of materials, the 6th, 8th and 10th SH coefficients for this group are all very small. These differences in the configurations of the $\mathrm{SH}$ coefficient amplitudes contribute to the completely different single crystal Young's modulus surfaces for these two group of materials, as is evident in [4].

\subsubsection{The computational model and the pseudo-kernel}

In practice, ultrasonic waves are typically excited into a crystalline bulk from a transducer and received from different locations to investigate the wave velocities in various directions. In this paper, however, we wish to establish a proof of principle for the generalised theorem as we did for the theorem for HCP polycrystals in a previous paper [1], so that simulated velocities for a few representative experimentally obtained cubic textures will be utilized, instead of experimentally measured wave velocities, to evaluate the accuracies of forward wave speed predictions as well as to inversely extract the ODF information.

In order to generate the simulated velocities for such analyses, the Representative Volume Element (RVE) finite element model established in [18] in Abaqus/Explicit is utilized again. As shown in 
Figure 4a, the model was finely-meshed to simulate the ultrasonic wave propagation in polycrystalline aggregates. There were in total 2160 grains in the model, and the elastic properties of the grains were specified to take full account of the crystallographic orientation of every grain resulting from the elastic anisotropy. By doing so a range of differing crystallographic textures could be studied, and the overall ultrasonic wave speeds for the textures could be determined (note that the RVE model only accounts for the overall crystallographic texture which is the predominant affecting factor for transmission wave speeds, and discards morphologies and grain boundaries etc, as argued in [1] ).

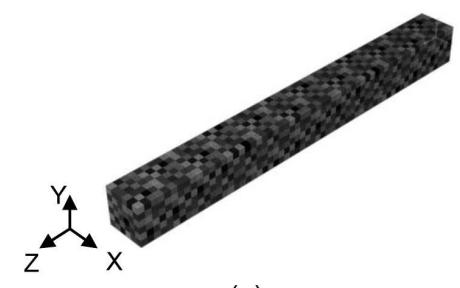

(a)

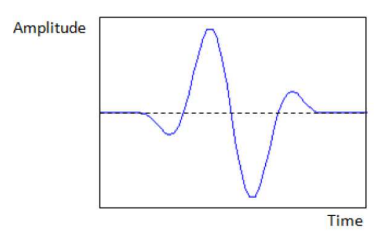

(b)

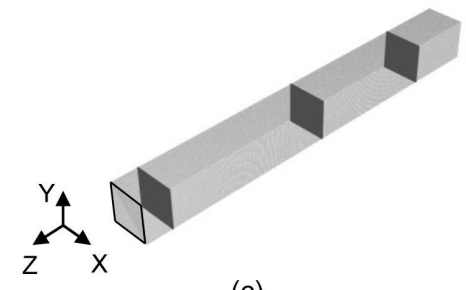

(c)

Figure 4: a. The RVE model containing 2160 crystals; b. ultrasonic wave loaded as sinusoidal pulses in Hanning window; c. planes in the model for wave loading and data extraction [18].

The ultrasonic waves propagating through the model were applied as pressure waves on the near-end of the model, with their amplitudes changing as sinusoidal pulses in a Hanning window, as shown by Figure $4 \mathrm{~b}$ [18]. The centre frequency of the pulses is $2 \mathrm{MHz}$, making the wavelength about twice the size of a crystal. The four lateral faces of the bar are all fixed in normal directions, enabling the waves applied on the near-end and propagating along the bar to be considered as plane waves. As the waves advance they pass a number of planes shown in Figure 4c, on which stresses and strains were tracked such that the arrival times were detected and the average wave velocities were calculated.

It needs to be stressed that as discussed in Section 2.2 and shown in the Appendix, the particle displacement direction can deviate significantly from the phase velocity direction along the bar. While this angular difference may be pre-calculated and compensated for single crystal simulations by carefully setting the boundary conditions, it is normally not possible for polycrystals where the particle displacement direction varies at each point along the propagation route and cannot be predetermined. Therefore the plane wave condition does not hold any more and dispersion and dissipation come into play, resulting in smaller simulated phase velocities obtained compared to the theoretical ones.

A practical way is adopted in the following parts of the paper to avoid this computational error affecting our studies. Whilst being aware that the actual phase velocities should be the solutions to the Christoffel equation and that the SH kernels shown in Tables 3 and 4 should be used in experimental tests, we instead use the single crystal SH coefficients obtained via the simulation model with normal boundary conditions consistent with all subsequent simulations on polycrystals as the pseudo-kernel in the generalised convolution theorem to perform both forward and inverse studies. By virtue of the consistency of the boundary conditions, this serves as a verification of the theorem. Iron is used as the exemplary material for these demonstrations, and its pseudo-kernel coefficients to be used in all later studies are shown in contrast with the original ones in Table 5.

\subsection{Theoretical orientation distributions}

A single crystal satisfies our assumption of homogeneity in deduction of the generalised theorem such that when evaluating from any angles in the space, the waves always go through the same crystal, so 
Table 5: Original and pseudo-kernel SH coefficients for single crystal Fe

\begin{tabular}{ccccccc}
\hline \hline & $K_{00}$ & $K_{20}$ & $K_{40}$ & $K_{60}$ & $K_{80}$ & $K_{10,0}$ \\
\hline Original & 21303.6 & 0.0 & -611.8 & -4.2 & -52.2 & -2.2 \\
Pseudo & 20982.2 & 0.0 & -686.5 & -6.8 & 73.9 & -2.2 \\
\hline \hline
\end{tabular}

the theorem stands in this case. Here, some simple orientations on a single crystal Fe are considered as demonstrations.

It has already been demonstrated in Section 3.3.4 that $W_{l m 0}$ with $m \geq 0$ contain all the linearly independent terms needed to reconstruct the original ODF matrices. Therefore in the following parts of the paper, only these coefficients will be shown when referring to an ODF.

\subsubsection{Single crystal with reference configuration}

The reference configuration is the simplest case for a single crystal, with the crystal system oriented exactly the same as the sample system. The ODF for this case is therefore a Dirac impulse at $\alpha=\beta=\gamma=0$, or in fact a series of impulses at all equivalent orientations with the symmetries considered. So the ODF coefficients $W_{l m n}$ can be then calculated accordingly by inputting all the orientations into equation 15 .

Then to verify the generalised theorem, these $W_{l m n}$ are input into equation 19 to obtain the resulting polycrystal velocity SH coefficients $V_{l m}$. All the calculated non-zero $W_{l m 0}$ as well as the resulting $V_{l m}$, both of $l \leq 10$ and $m \geq 0$, are listed in Table 6 , and the other non-zero coefficients can be determined using the relationships listed in Table 2.

For the reference configuration, the resulting velocity surface is expected to be exactly the same as

Table 6: Single crystal with reference configuration

\begin{tabular}{|c|c|c|c|}
\hline \multicolumn{4}{|l|}{$W_{000}=1$} \\
\hline$W_{400}=0.5833$ & $W_{440}=0.3486$ & & \\
\hline$W_{600}=0.125$ & $W_{640}=-0.2339$ & & \\
\hline$W_{800}=0.5785$ & $W_{840}=0.2175$ & $W_{880}=0.3315$ & \\
\hline$W_{10,0,0}=0.1693$ & $W_{10,4,0}=-0.1706$ & $W_{10,8,0}=-0.2030$ & Kernel surface plot \\
\hline \multicolumn{4}{|l|}{$V_{00}=21303.6$} \\
\hline$V_{40}=-611.8$ & $V_{44}=-365.6$ & & \\
\hline$V_{60}=-4.2$ & $V_{64}=7.9$ & & \\
\hline$V_{80}=-52.2$ & $V_{84}=-19.6$ & $V_{88}=-29.9$ & \\
\hline$V_{10,0}=-2.2$ & $V_{10,4}=2.2$ & $V_{10,8}=2.6$ & Rotated surface plot \\
\hline
\end{tabular}

Note that only $W_{l m 0}$ are listed here and in the following figures, and that the other non-zero coefficients are linearly dependent on $W_{l m 0}$

the kernel, which is demonstrated in Table 6, with the resulting SH coefficients of the polycrystal velocity function after convolving the ODF coefficients with the kernel being exactly the same as the kernel coefficients. It is also noticeable that for this special orientation, $W_{l m 0}$ has non-zero values only when $m=4 k$. 


\subsubsection{Single crystal oriented with $\alpha=\pi / 4$}

Now consider a single cubic crystal with an orientation rotated from the reference configuration, for example, with $\alpha=\frac{\pi}{4}$ and $\beta=\gamma=0$. Similarly with the reference configuration, the ODF is a series of Dirac impulses at all the equivalent orientations to $(\pi / 4,0,0)$ given by the crystal symmetries, and the coefficients can then be calculated accordingly. The non-zero values of the $W_{l m n}$ and the $V_{l m}$ obtained after convolution, both of $l \leq 10$ and $m \geq 0$, are listed in Table 7 .

With $\alpha$ being $\pi / 4$ and $\beta, \gamma$ both 0 , the only expected difference made to the kernel is to rotate it

Table 7: Single crystal with $\alpha=\pi / 4$

\begin{tabular}{|c|c|c|c|}
\hline \multicolumn{4}{|l|}{$W_{000}=1$} \\
\hline$W_{400}=0.5833$ & $W_{440}=-0.3486$ & & \\
\hline$W_{600}=0.125$ & $W_{640}=0.2339$ & & \\
\hline$W_{800}=0.5785$ & $W_{840}=-0.2175$ & $W_{880}=0.3315$ & \\
\hline$W_{10,0,0}=0.1693$ & $W_{10,4,0}=0.1706$ & $W_{10,8,0}=-0.2030$ & Kernel surface plot \\
\hline \multicolumn{4}{|l|}{$V_{00}=21303.6$} \\
\hline$V_{40}=-611.8$ & $V_{44}=365.6$ & & \\
\hline$V_{60}=-4.2$ & $V_{64}=-7.9$ & & \\
\hline$V_{80}=-52.2$ & $V_{84}=19.6$ & $V_{88}=-29.9$ & \\
\hline$V_{10,0}=-2.2$ & $V_{10,4}=-2.2$ & $V_{10,8}=2.6$ & Rotated surface plot \\
\hline
\end{tabular}

about the $Z$-axis by the angle of $\pi / 4$, and the figures in Table 7 shows exactly the same result as expected. As for the ODF coefficients, this rotated orientation only changes the signs of the $W_{l 40}$, which results as the change of signs of the convolved velocity coefficients $V_{l 4}$, which then eventually cause the velocity surface to be rotated about the $Z$-axis in the reconstruction of the surface.

\subsubsection{Single crystal oriented with $\alpha=\beta=\gamma=\pi / 4$}

Next, consider a slightly more complicated single cubic crystal orientation, with $\alpha=\beta=\gamma=\pi / 4$. The calculation of the coefficients is still the same as the previous orientations, with the ODF being impulses at all equivalent orientations to $(\pi / 4, \pi / 4, \pi / 4)$. Some of the the non-zero coefficients $W_{l m n}$ and $V_{l m}$ are also listed in Table 8 . However, only those with $l \leq 8$ are listed here for consideration of space.

For this case, the single crystal is rotated firstly about the $Z$-axis for $\pi / 4$, then about the rotated $Y^{\prime}$-axis for $\pi / 4$, and finally about the rotated $Z^{\prime \prime}$ for another $\pi / 4$. As a result, the (111) vector of the crystal system should be along the (001) direction in the sample system (gray box in the figures) and (001) in the crystal system along the $(1,1, \sqrt{2})$ direction. The figures in Table 8 show that this rotation is done correctly with this group of $W_{l m n}$.

One difference of this orientation compared to the previous two, however, is that many more ODF coefficients are finite, with all $W_{l m 0}$ of $-l \leq m \leq l$ now having non-zero values, which then results in all the convolved velocity $V_{l m}$ being non-zero.

For these respective single crystal orientations, the theorem is demonstrated to have applied correct rotations on the velocity surfaces, and the underlying $\mathrm{SH}$ coefficients for these cases are also calculated. It is notable that even for these single crystal orientations, which are the strongest textures possible, 
Table 8: Single crystal with $\alpha=\beta=\gamma=\pi / 4$

\begin{tabular}{|c|c|c|c|}
\hline \multicolumn{3}{|l|}{$W_{000}=1$} & \multirow{9}{*}{ Kernel surface } \\
\hline$W_{400}=-0.328$ & $W_{410}=0.115+0.115 i$ & $W_{420}=0.115 i$ & \\
\hline$W_{430}=-0.305+0.305 i$ & $W_{440}=0.109$ & & \\
\hline$W_{600}=0.166$ & $W_{610}=-0.063-0.063 i$ & $W_{620}=0.090 i$ & \\
\hline$W_{630}=-0.070+0.070 i$ & $W_{640}=0.048$ & $W_{650}=-0.036-0.036 i$ & \\
\hline \multicolumn{3}{|l|}{$W_{660}=-0.134 i$} & \\
\hline$W_{800}=0.050$ & $W_{810}=-0.103-0.103 i$ & $W_{820}=-0.135 i$ & \\
\hline$W_{830}=0.129-0.129 i$ & $W_{840}=-0.225$ & $W_{850}=-0.089-0.089 i$ & \\
\hline$W_{860}=-0.301 i$ & $W_{870}=-0.114+0.114 i$ & $W_{880}=0.029$ & \\
\hline \multicolumn{3}{|l|}{$V_{00}=20982.2$} & \multirow{9}{*}{ Rotated surface } \\
\hline$V_{40}=386.2$ & $V_{41}=-135.7-135.7 i$ & $V_{42}=-135.7 i$ & \\
\hline$V_{43}=359.0-359.0 i$ & $V_{44}=-128.2$ & & \\
\hline$V_{60}=-9.0$ & $V_{61}=3.4+3.4 i$ & $V_{62}=-4.9 i$ & \\
\hline$V_{63}=3.8-3.8 i$ & $V_{64}=-2.6$ & $V_{65}=2.0+2.0 i$ & \\
\hline \multicolumn{3}{|l|}{$V_{66}=7.2 i$} & \\
\hline$V_{80}=-7.2$ & $V_{81}=-14.8-14.8 i$ & $V_{82}=-19.3 i$ & \\
\hline$V_{83}=18.4-18.4 i$ & $V_{84}=-32.2$ & $V_{85}=-12.7-12.7 i$ & \\
\hline$V_{86}=-43.1 i$ & $V_{87}=-16.3+16.3 i$ & $V_{88}=4.1$ & \\
\hline
\end{tabular}

the 10th degree SH coefficients of $V_{l m}$ are smaller than 2.6, which means the contributions of the ODF to $V_{l m}$ and $V_{10 m}$ to the velocity surface are both small and negligible. Therefore in the following, focus is given only to coefficients with $l \leq 8$.

\subsection{Studies on experimental polycrystal textures}

\subsubsection{Textures to be investigated}

It has been demonstrated in the previous section how to apply the rotations on single cubic crystals through equation 19 and therefore validated the convolution theorem; but in reality, the textures in polycrystalline aggregates are much more complex. However, provided the ODF of the texture is known, forward studies can be carried out in a similar fashion to predict the polycrystal wave velocities using equation 19.

Here, the subjects of our forward studies are the three experimental representative cubic textures illustrated in Figure 5, with the two figures in each row showing respectively the (001) and (111) pole figure plots of the same texture. Details of the forward studies are fully demonstrated as follows: firstly, from the texture information, the corresponding sets of ODF coefficients are determined, which are then convolved with the kernel to calculate the $\mathrm{SH}$ coefficients of the polycrystal velocity function; finally, these coefficients are employed by means of SH expansions to reconstruct the velocity surfaces, which are then plotted, and the polycrystal velocities in the three-dimensional space are predicted. 

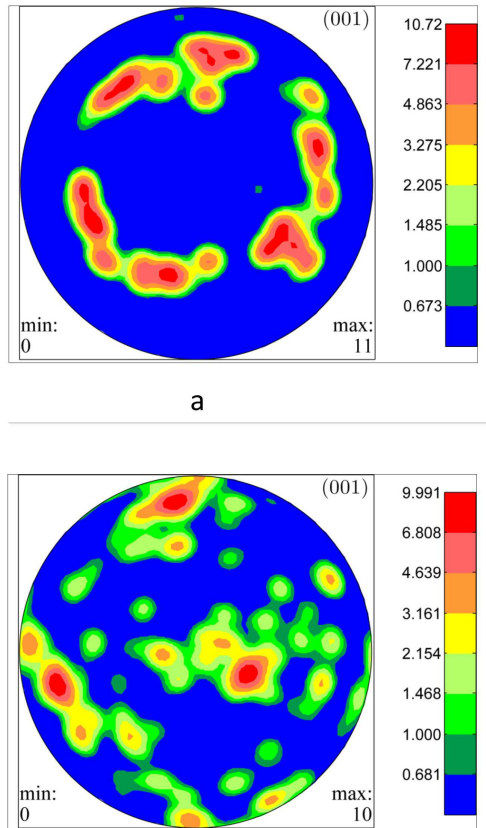

C

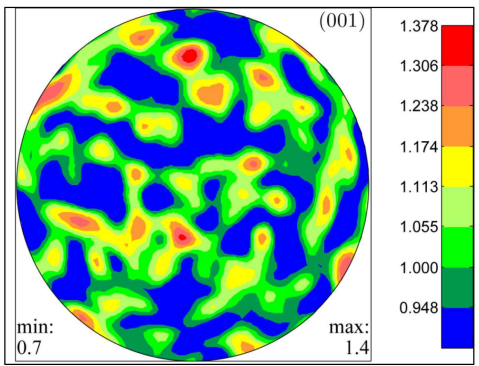

e

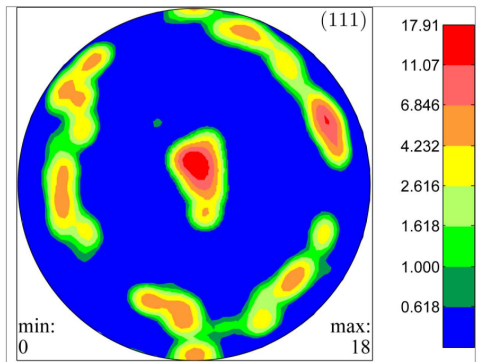

b

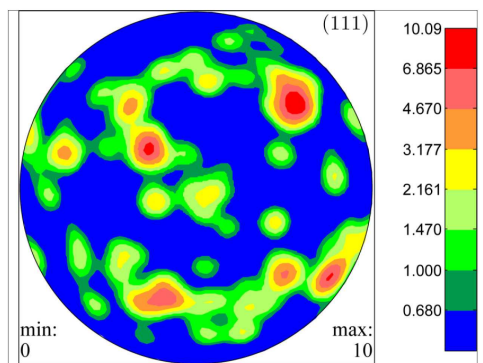

d

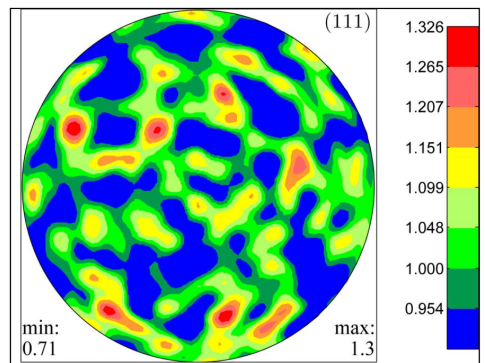

Figure 5: The pole figure plots of the three representative experimental cubic textures to be investigated, with a and b: the (001) and (111) pole figures of Texture-1, respectively; c and d: the pole figures of Texture-2; and e and f: the pole figures of Texture-3.

Through the study and the results obtained, the relationship between the ODF and the polycrystalline velocities are further explored.

\subsubsection{The forward study routine and results of Texture-1}

It can be seen from Figure 5 that the texture shown by the first row, labelled Texture-1, has the cubic crystals predominantly aligned with their (111) reciprocal vectors along the direction of the north pole, which is also the normal direction of the sample. The orientation distributions about the north pole, however, are not significantly preferred, as shown by the circle about the centre in the (001) pole figure. There are some clusters on the circle, but these are not strong.

The ODF coefficients of the texture can be readily calculated with the aid of the MTEX toolbox for Matlab [25], and the non-zero independent ones are listed in the first half of Table 9. The SH coefficients of the polycrystal velocity function are then obtained through the matrix-vector multiplication described by equation 19 using the calculated ODF coefficients, and the results are shown in the second half of Table 9 . 
Table 9: The coefficients for the ODF and the resulting polycrystal velocity surface of Texture-1

\begin{tabular}{|c|c|c|}
\hline \multicolumn{3}{|l|}{$W_{000}=1.0$} \\
\hline$W_{400}=-0.291$ & $W_{410}=-0.0771+0.0099 i$ & $W_{420}=0.0141+0.0090 i$ \\
\hline$W_{430}=0.1034+0.0569 i$ & $W_{440}=0.00523+0.00300 i$ & \\
\hline$W_{600}=0.127$ & $W_{610}=0.0488-0.0311 i$ & $W_{620}=0.0351+0.0032 i$ \\
\hline$W_{630}=0.0283+0.0132 i$ & $W_{640}=0.00784-0.00267 i$ & $W_{650}=-0.0008+0.0184 i$ \\
\hline \multicolumn{3}{|l|}{$W_{660}=0.0228-0.0401 i$} \\
\hline$W_{800}=0.0431$ & $W_{810}=0.0339+0.0195 i$ & $W_{820}=-0.00948-0.00299 i$ \\
\hline$W_{830}=-0.0454-0.0312 i$ & $W_{840}=0.0069-0.0243 i$ & $W_{850}=-0.0181+0.0462 i$ \\
\hline$W_{860}=0.0249-0.0814 i$ & $W_{870}=0.0025-0.0441 i$ & $W_{880}=0.01022-0.00408 i$ \\
\hline \multicolumn{3}{|l|}{$V_{00}=20982.2$} \\
\hline$V_{40}=342.3$ & $V_{41}=90.7-11.7 i$ & $V_{42}=-16.6-10.6 i$ \\
\hline$V_{43}=-121.7-67 . i$ & $V_{44}=-6.2-3.5 i$ & \\
\hline$V_{60}=-6.9$ & $V_{61}=-2.6+1.7 i$ & $V_{62}=-1.9-0.2 i$ \\
\hline$V_{63}=-1.5-0.7 i$ & $V_{64}=-0.4+0.1 i$ & $V_{65}=0 .-1 . i$ \\
\hline \multicolumn{3}{|l|}{$V_{66}=-1.2+2.2 i$} \\
\hline$V_{80}=6.2$ & $V_{81}=4.9+2.8 i$ & $V_{82}=-1.4-0.4 i$ \\
\hline$V_{83}=-6.5-4.5 i$ & $V_{84}=1 .-3.5 i$ & $V_{85}=-2.6+6.6 i$ \\
\hline$V_{86}=3.6-11.7 i$ & $V_{87}=0.4-6.3 i$ & $V_{88}=1.5-0.6 i$ \\
\hline
\end{tabular}

Note that only $W_{l m 0}$ and $V_{l 0}$ are listed here, and that the other non-zero coeffs are linearly dependent on them.

The polycrystal velocity surface band-limited up to the 8th degree can be recovered using the obtained SH coefficients:

$$
v(\theta, \phi)=\sum_{l=0}^{8} \sum_{n=-l}^{l} V_{l m} Y_{l m}(\theta, \phi)
$$

The reconstructed velocity surface for Texture-1 is plotted in Figure 6, with Figure 6a illustrating the velocity variations as three-dimensional shape changes and Figure $6 \mathrm{~b}$ showing the stereographic projection of its upper half onto the equator in a similar way as for pole figures. It can be seen that because of the preferred (111) pole distributions along the north pole and the fact that the (111) direction has the largest wave speed within a single crystal, the polycrystal velocities are also the largest near the north pole; and because the (111) poles on the peripheries of the figure are evenly distributed, the velocities near the equator also display very close amplitudes. The blue regions in the middle of Figure $6 \mathrm{~b}$ are resulting from the (001) pole distributions because the single crystal velocities along this pole are the smallest.

\subsubsection{Results of the other two textures}

The other two textures are studied using the same method. As for the previous texture, Texture-2 is also strongly textured according to the intensities of its pole figures in the second row of Figure 5. 

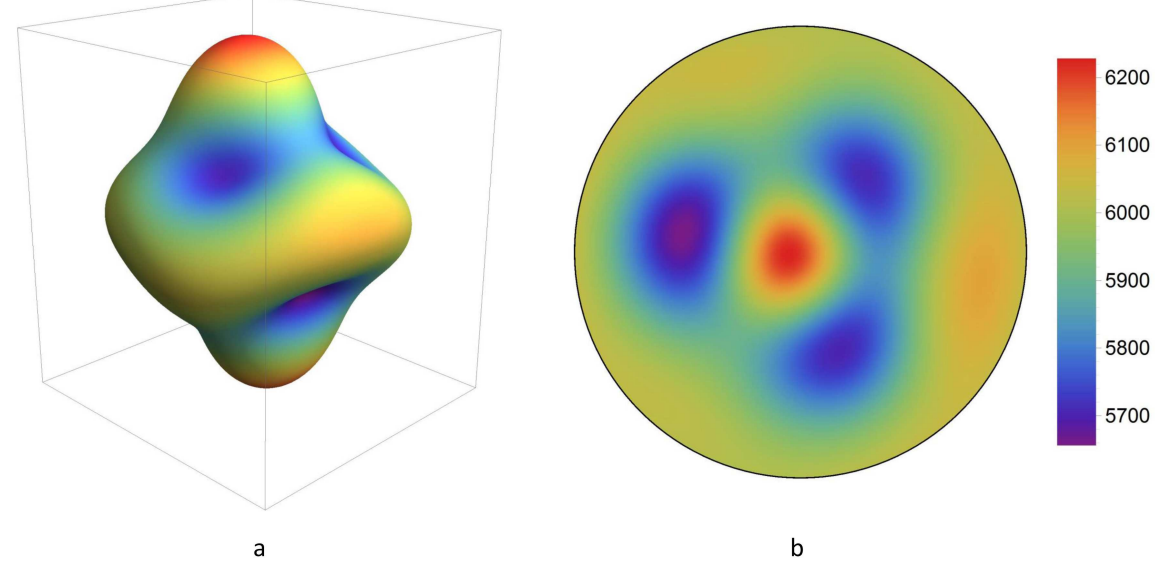

Figure 6: The polycrystal velocity surface of Texture-1, shown as: a. three-dimensional shape; b. the stereographic projection of the upper part onto the equator.

The red spots in its (001) pole figure, as well the four preferred orientations in the (111) pole figure, suggest that the texture is in loose approximation with a rotated single crystal.

Similarly, the ODF coefficients are output and polycrystal velocity coefficients are calculated. But here, only the latter are shown for space reasons. The values for Texture- 2 are listed in Table 10, and the reconstructed velocity surface is also plotted in two ways in Figure 7.

Table 10: The polycrystal velocity SH coefficients calculated from the ODF of Texture-2

\begin{tabular}{llll}
\hline \hline$V_{00}=20982.2$ & & & \\
\hline$V_{40}=-72.1$ & $V_{41}=35.1+98.7 i$ & $V_{42}=67.8+7.5 i$ & $V_{43}=-80.4-26.9 i$ \\
$V_{44}=-90.6-65.9 i$ & & & \\
\hline$V_{60}=-2.6$ & $V_{61}=-0.1+0.2 i$ & $V_{62}=1.4-1.7 i$ & $V_{63}=0.6-1.3 i$ \\
$V_{64}=1.1-0.1 i$ & $V_{65}=0.1-1.4 i$ & $V_{66}=-0.7-0.5 i$ & \\
\hline$V_{80}=-4.6$ & $V_{81}=-1.6$ & $V_{82}=-5.1+0.5 i$ & $V_{83}=3.6-1 . i$ \\
$V_{84}=-0.3-5.1 i$ & $V_{85}=0.4-2.1 i$ & $V_{86}=-0.2$ & $V_{87}=4.3+2.7 i$ \\
$V_{88}=1.1+1.1 i$ & & & \\
\hline \hline
\end{tabular}

Figure 7a shows that the polycrystal velocity surface of Texture- 2 is indeed approximately in the shape of a distorted and rotated cube, which agrees with the information from the pole figures.

The last texture, labelled Texture-3 and shown by the last row of pole figures in Figure 5, has near theoretically random orientation distributions and therefore almost uniform intensities in all the pole figures, suggesting that the texture may be from a powder-metallurgy sample. The resulting SH coefficients of the polycrystal velocity surface are similarly calculated and listed in Table 11, and the surface itself is plotted in Figure 8.

Theoretically, a polycrystal aggregate with random texture should be elastically isotropic, so the wave velocities should be approximately the same regardless of the incident direction. It is shown in 

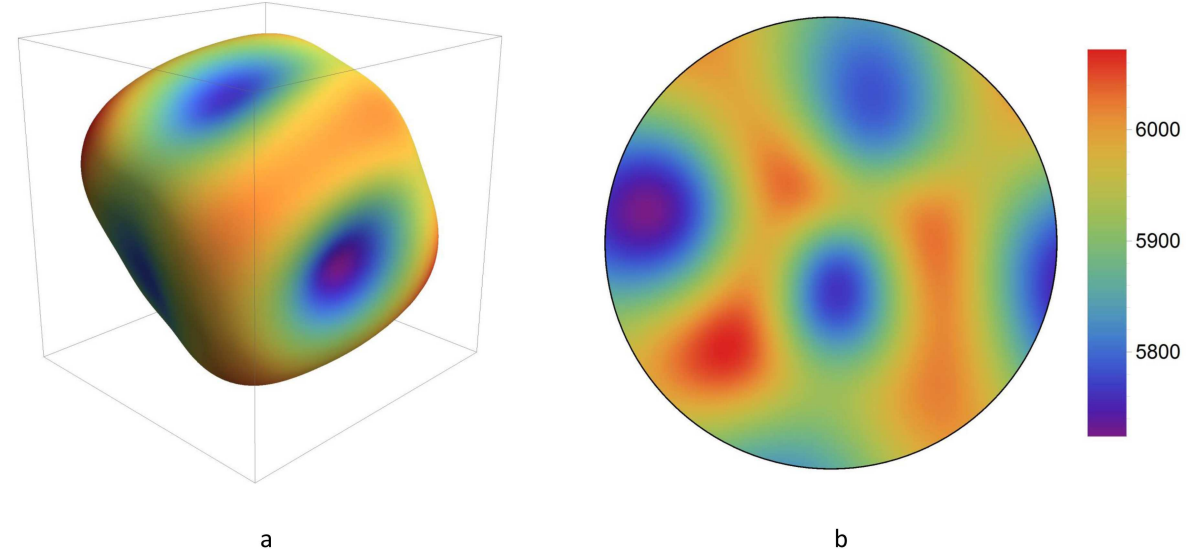

Figure 7: The polycrystal velocity surface of Texture-2, shown as: a. three-dimensional shape; b. the stereographic projection of the upper part onto the equator.

Table 11: The polycrystal velocity SH coefficients calculated from the ODF of Texture-3

\begin{tabular}{llll}
\hline \hline$V_{00}=20982.2$ & & & \\
\hline$V_{40}=0.9$ & $V_{41}=4.6-0.3 i$ & $V_{42}=-2.1+4.6 i$ & $V_{43}=-1.2-0.9 i$ \\
$V_{44}=3.9-0.4 i$ & & & \\
\hline$V_{60}=0$. & $V_{61}=0.1$ & $V_{62}=0$. & $V_{63}=-0.1$ \\
$V_{64}=0$. & $V_{65}=-0.1+0.1 i$ & $V_{66}=0 .-0.1 i$ & \\
\hline$V_{80}=0.1$ & $V_{81}=0 .-0.2 i$ & $V_{82}=-0.3-0.1 i$ & $V_{83}=-0.4+0.7 i$ \\
$V_{84}=-0.2-0.1 i$ & $V_{85}=0.1-0.4 i$ & $V_{86}=0.1-0.1 i$ & $V_{87}=0.3+0.3 i$ \\
$V_{88}=-0.2+0.4 i$ & & & \\
\hline \hline
\end{tabular}

Figure $8 \mathrm{a}$ that the velocity surface is indeed close to a sphere, and the largest difference in velocity amplitudes is no more than $20 \mathrm{~m} / \mathrm{s}$, as shown by Figure $8 \mathrm{~b}$.

So far we have successfully performed forward studies on the three experimental textures and reconstructed the polycrystal velocity surface, which all qualitatively agree with the pole figures. These results will be compared to independent simulation results in following sections to show quantitatively the correctness and accuracy of the generalised convolution technique.

\subsection{Discussions of the forward studies}

The forward studies on theoretical distributions and experimental textures have revealed the following properties about the ODF and velocity surfaces themselves and the relationships between them. First, for general cases (eg the single crystal orientation with $\alpha=\beta=\gamma=\pi / 4$ or indeed all the experiential textures), many terms of the ODF and the velocity surface coefficients are complex numbers, and omitting the imaginary parts $[14,16]$ leads to errors in both the amplitudes and phases in the ODF and reconstructed velocity surfaces. 

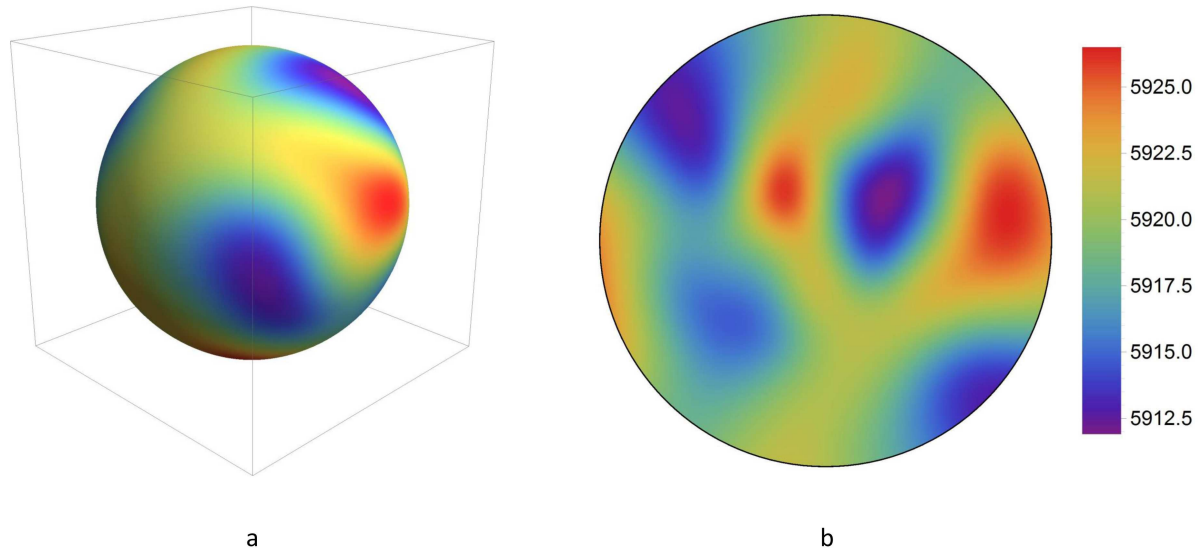

Figure 8: The polycrystal velocity surface of Texture-3, shown as: a. three-dimensional shape; b. the stereographic projection of the upper part onto the equator.

Secondly, for the material Fe, the polycrystal velocity SH coefficients $V_{l m}$ of the degrees $6 \sim 10$ are all less than 10 for all the experimental textures, even though two of them are strongly textured. This is because the ODF coefficients $W_{l m n}$ of the corresponding degrees are very small (partly resulting from the $1 /(2 l+1)$ term in equation 21$)$, so despite that the 8 th degree coefficient of the kernel $K_{80}$ has relatively large amplitude, the resulting $V_{l m}$ after convolving the kernel with the ODF are still small. This means that when reconstructing the polycrystal velocity surfaces, the contributions from $V_{l m}$ of $6 \sim 10$ th degrees to the overall velocities are no more than $10 \mathrm{~m} / \mathrm{s}$ respectively, which are in the same order with the experimental errors of ultrasonic tests and hence can be neglected. As the $V_{l m}$ are obtained through the convolution process, these contributions are in fact from the ODF coefficients of these degrees.

This leads to two more important implications: first, it demonstrates that the ultrasonic wave velocities in these cubic materials only have strong dependence on the 4th degree ODF coefficients, and the contributions from the higher degree coefficients are relatively weak and of negligible amplitudes of less than 10. So when performing the forward studies to predict the polycrystal velocities, the theoretical accuracy of approximately $\pm 20 \mathrm{~m} / \mathrm{s}$ can be achieved by using the coefficients up to the 4th degree only. Second and more importantly, it is therefore not possible to retrieve the ODF coefficients of 6th degree or higher using ultrasonic wave tests, because accurate results of the velocity coefficients $V_{l m}$ are required in order to retrieve these ODF coefficients, which is not possible to achieve as their amplitudes are in the same order with experimental errors.

It also should be noted that even though the above conclusions are based on the elastic properties of the metal Fe, it applies to the group of materials with similar single crystal velocity surfaces with $\mathrm{Fe}$, including $\mathrm{Cu}, \mathrm{Ni}$, etc; it also applies for the other group of materials including $\mathrm{Al}, \mathrm{Nb}, \mathrm{V}$, etc, whose 6th, 8th and higher degree single crystal SH coefficients are even smaller compared to the Fe group, which results in even less contributions to the polycrystal velocities from the ODF coefficients of these degrees. 


\section{Inverse studies: from wave velocities to the ODF}

The generalised spherical convolution theorem has been employed in the previous section in forward studies to predict velocities from the texture information for both theoretical and experimental orientation distributions. In this section, it is to be used in the inverse sense to extract texture information from ultrasonic wave velocities, ie, to perform inverse studies.

\subsection{The Gaussian-Legendre quadrature scheme to obtain $V_{l m}$}

The direct objective of the inverse studies is the determination of the ODF coefficients, which would ultimately enable us to reconstruct the original ODF through the convolution expansion theory. As shown by the generalised theorem in equation 19, the ODF is convolved with the single crystal wave velocity to make the polycrystal velocity function and therefore may be obtained by de-convolving the kernel from the polycrystal velocities. In other words, in order to extract the texture information from ultrasonic tests, the first step is to establish the polycrystal velocity coefficients $V_{l m}$ from the velocity results.

The coefficients $V_{l m}$ are defined by projecting the velocity function $v(\theta, \phi)$ onto the complex conjugate of the corresponding spherical harmonic bases:

$$
V_{l m}=\int_{0}^{2 \pi} \int_{0}^{\pi} v(\theta, \phi) Y_{l m}^{*}(\theta, \phi) \sin (\theta) d \theta d \phi
$$

In practice, however, it is often not possible to perform continuous data extraction for the integration, but more often desirable to obtain the coefficients from a number of discrete data. The latter can be achieved by means of numerical integrations, and the choices of positions to obtain the discrete data are called quadratures. Comparisons of the two most widely used quadrature, namely equiangular and Gaussian-Legendre, were presented in the literature [18,27,28], and in this paper we continue to choose the latter for its ability to get to $(2 N-1)$ th degree of accuracy with only $2 N \times N$ discrete data. The numerical integration is written:

$$
V_{l m}=\frac{\pi}{N} \sum_{i=0}^{2 N-1} \sum_{j=0}^{N-1} v\left(\theta_{i}, \phi_{j}\right) Y_{l m}^{*}\left(\theta_{i}, \phi_{j}\right) \omega_{N}(i)
$$

where $\theta_{i}$ is the $i$ th Gaussian node for the polar angle, $\phi_{j}$ is equal to $(j-1) \cdot \pi / N$ to ensure the azimuthal angle goes equally spaced from 0 to $2 \pi$, and $\omega_{N}(i)$ is the well-known weight function for the $i$ th Gaussian node.

The same technique described in our previous paper [1] (which is referred to for a clearer explanation of the technique) is used here to generate the velocity at the particular nodes $v\left(\theta_{i}, \phi_{j}\right)$ using the RVE model introduced in Section 4.1.2. Instead of rotating the ultrasonic wave incident directions, it is the crystallographic orientations of all crystals in the model that are rotated systematically and correspondingly, with both the polycrystal model and the wave propagation direction fixed. Here evaluating the wave velocity in various different directions $\left(\theta_{i}, \phi_{j}\right)$ with respect to the sample system $o-x y z$ is equivalent to always evaluating the velocity in a fixed direction but with the sample system rotated by $\left(-\theta_{i},-\phi_{j}\right)$. The rotation of the sample system then causes the configurations of all the crystals in the model to undergo the same additional rotation, which may be calculated by 
left-multiplying the following rotation matrix:

$$
R=\left(\begin{array}{ccc}
\cos \theta_{i} \cos \phi_{j} & -\cos \theta_{i} \sin \phi_{j} & \sin \theta_{i} \\
\sin \phi_{j} & \cos \phi_{j} & 0 \\
-\sin \theta_{i} \cos \phi_{j} & \sin \theta_{i} \sin \phi_{j} & \cos \theta_{i}
\end{array}\right)
$$

The 4th degree of accuracy is desired in our studies, so theoretically at least $10 \times 5$ discrete data are needed for the Gaussian-Legendre quadrature. In the following part we choose $N=10$ to achieve both accuracy and efficiency.

\subsection{Simulated wave velocity surfaces \& retrieved ODF coefficients}

The three experimental textures in Figure 5 are all input to the RVE model and rotated to the corresponding configurations, taking full account of their crystallographic orientations and the additional rotation introduced by the Gaussian-Legendre quadrature scheme, as shown by equation 29. The computational results are then used to calculate the SH coefficients $V_{l m}$ of the polycrystal velocity function through equation 28 , and the resulting $V_{l m}$ may be used in two ways: on one hand, they can be utilized to reconstruct the velocity surfaces, which, when compared with the velocity surfaces predicted by the generalised convolution theorem in Section 4.3, enable us to verify the accuracy of the theorem in the forward studies; on the other hand, the ODF information contained in the calculated $V_{l m}$ may be obtained by de-convolving the SH coefficients of single crystal velocity function out from the $V_{l m}$.

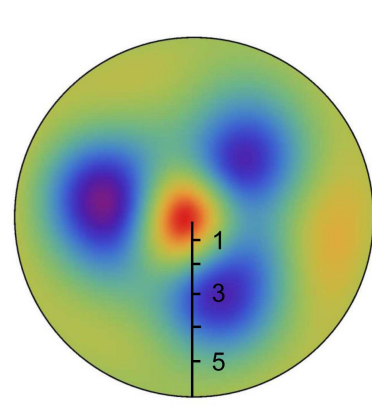

a

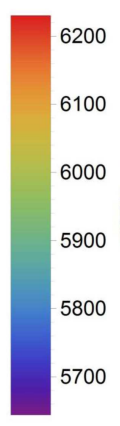

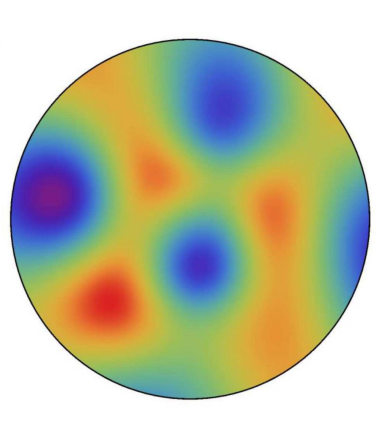

b

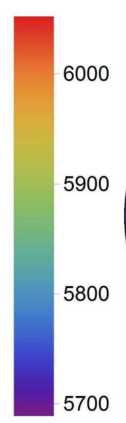

5700

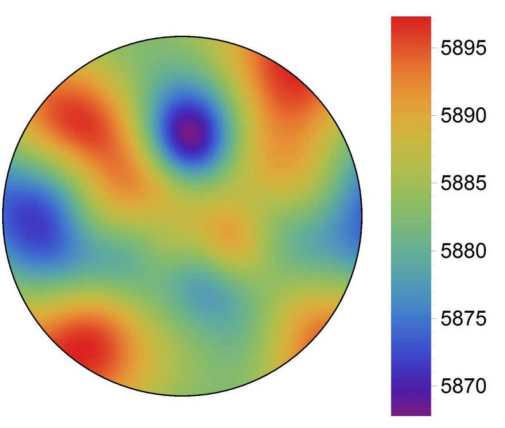

C

Figure 9: The stereographic projections of the polycrystal velocity surfaces obtained via the simulation method, with a, b, c corresponding respectively to Texture-1, 2, 3. Note that the different appearance of plot c with Figure 8b results from their fine colourscales and that the near-isotropy in wave speed for Texture-3 is well reflected in both plots.

The reconstructed polycrystal velocity surfaces from the $V_{l m}$ are plotted in Figure 9 , with a, b, c corresponding to the simulation results of Texture-1, 2, 3 in Figure 5, respectively. It can be seen that for Texture-1 and 2 whose textures are strongly preferred and elastic anisotropies are pronounced, the results agree very well with the predictions of the theorem in Figures 6 and 7 . However, for Texture-3 whose orientation distribution is almost random, the contour results seem rather different to the predictions in Figure 8. This is because the random texture results in very small differences in the polycrystal spatial velocities (about $10 \mathrm{~m} / \mathrm{s}$ in this case), which are the same level as the errors of the simulation model(and of the ultrasonic wave experiment techniques too), so that the model velocity differences are difficult to extract. Nevertheless, the simulation results do capture the most 
important characteristic of the velocity surface for the random texture; that is, velocities are almost the same regardless of the incident direction, with largest difference less than $20 \mathrm{~m} / \mathrm{s}$.

Table 12: Comparison between the predicted and simulated velocities of Texture-1 at 5 Gaussian nodes

\begin{tabular}{cccccc}
\hline \hline Gaussian nodes $\left(\theta_{i}, 0\right)$ & $\mathrm{i}=1$ & $\mathrm{i}=2$ & $\mathrm{i}=3$ & $\mathrm{i}=4$ & $\mathrm{i}=5$ \\
\hline Predicted $(\mathrm{m} / \mathrm{s})$ & 6074.3 & 5868.5 & 5854.5 & 6013.2 & 6088.7 \\
Simulation $(\mathrm{m} / \mathrm{s})$ & 6044.3 & 5849.7 & 5845.3 & 5990.1 & 6065.0 \\
\hline
\end{tabular}

It is also noticeable that for the Texture-3, even though both the simulation results and the predictions display no significant velocity changes with direction, the amplitudes of the former are smaller compared to the latter. Indeed, this is also the case for Texture-1 and 2 as well. Take the two sets of results at the five Gaussian nodes in Figure 9a for example, as listed in Table 12, the simulation results are also smaller than the predicted values. This phenomenon has been discussed in our previous paper [1] and can be explained in the same way: in the generalised theorem there are no inter-grain effects taken into consideration, and grains are treated as arrays of crystals. However, in the simulation model or experimental practice, neighbouring grains always have interactions on each other such that, when the waves propagates through neighbouring grains with different velocities, local stress fields are generated which eventually slow the waves down. Or in other words, the crystals in polycrystalline aggregates do not exhibit exactly the same properties as the theoretical kernel due to the confinement from neighbouring grains. In addition, because the elastic anisotropy of $\mathrm{Fe}$ is known to be more pronounced than $\mathrm{Ti}$, the differences between the two sets of velocities for Fe are a little larger than those of $\mathrm{Ti}[1]$.

We now move on to the inverse problem to perform the deconvolution on $V_{l m}$ and to extract the ODF coefficients. According to the relationships of $W_{4 m n}$ listed in Table 2 and similarly of $K_{4 n}$, the coefficients $W_{4 m 0}$ can be calculated via:

$$
W_{4 m 0}=\frac{V_{l m}}{\left(1+2 \times 0.59761430^{2}\right) K_{40}}, \text { where }-l \leq m \leq l .
$$

It has been mentioned that $W_{4 \bar{m} 0}$ and $W_{4 m 0}$ are complex conjugates to each other, hence only $W_{4 m 0}$ with $m \geq 0$, which are the only linearly independent coefficients, are listed in Table 13. It can be seen that for these textures, all the coefficients of the 4th degree including $W_{410}$ and $W_{430}$ are recovered. For Texture-1 and 2, the coefficients obtained via simulated velocities are of good accuracy compared to the original ones, with both the real and imaginary parts well retrieved; but the results of Texture-3 is less good. This results from the previously discussed issue of $V_{l m}$ being very small and beyond the accuracy of the simulation model. It is also directly related with the ODF, as shown by Table 13, the original ODF coefficients of the random texture are one or two orders smaller than the other two textures. This simply reflects that Texture-3 is, to all interests and purposes, random, and the small deviations from randomness cannot be nor need to be actually quantified.

\subsection{Recovering the ODF \& discussions}

The recovered 4th degree coefficients can be utilized to represent texture. The band-limited ODFs to the 4th degree are reconstructed via equation 14 using the coefficients listed in Table 13. The pole figures for the reconstructed ODFs are shown in Figure 10, in order to provide direct comparison with 
Table 13: The original and recovered 4th degree ODF coefficients of the three textures

\begin{tabular}{|c|c|c|c|}
\hline \multirow{2}{*}{ Texture-1 } & $\begin{array}{l}\text { Original ODF } \\
\text { coefficients }\end{array}$ & $\begin{array}{l}W_{400}=-0.291 \\
W_{420}=0.0141+0.0090 i \\
W_{440}=0.00523+0.00300 i\end{array}$ & $\begin{array}{l}W_{410}=-0.0771+0.0099 i \\
W_{430}=0.1034+0.0569 i\end{array}$ \\
\hline & $\begin{array}{l}\text { Recovered from } \\
\text { simulations }\end{array}$ & $\begin{array}{l}W_{400}=-0.278 \\
W_{420}=0.0145+0.0079 i \\
W_{440}=-0.00053+0.00415 i\end{array}$ & $\begin{array}{l}W_{410}=-0.0696+0.0035 i \\
W_{430}=0.1137+0.0587 i\end{array}$ \\
\hline \multirow{2}{*}{ Texture-2 } & $\begin{array}{l}\text { Original ODF } \\
\text { coefficients }\end{array}$ & $\begin{array}{l}W_{400}=0.0613 \\
W_{420}=-0.0576-0.0064 i \\
W_{440}=0.0770+0.0560 i\end{array}$ & $\begin{array}{l}W_{410}=-0.0298-0.0839 i \\
W_{430}=0.0683+0.0228 i\end{array}$ \\
\hline & $\begin{array}{l}\text { Recovered from } \\
\text { simulations }\end{array}$ & $\begin{array}{l}W_{400}=0.0476 \\
W_{420}=-0.0561-0.0014 i \\
W_{440}=0.0744+0.0557 i\end{array}$ & $\begin{array}{l}W_{410}=-0.0356-0.0871 i \\
W_{430}=0.0825+0.0201 i\end{array}$ \\
\hline \multirow{2}{*}{ Texture-3 } & $\begin{array}{l}\text { Original ODF } \\
\text { coefficients }\end{array}$ & $\begin{array}{l}W_{400}=-0.000769 \\
W_{420}=0.00180-0.00387 i \\
W_{440}=-0.00333+0.00030 i\end{array}$ & $\begin{array}{l}W_{410}=-0.00395+0.00024 i \\
W_{430}=0.001042+0.000731 i\end{array}$ \\
\hline & $\begin{array}{l}\text { Recovered from } \\
\text { simulations }\end{array}$ & $\begin{array}{l}W_{400}=-0.00424 \\
W_{420}=-0.00579-0.00294 i \\
W_{440}=0.00749-0.00186 i\end{array}$ & $\begin{array}{l}W_{410}=-0.000195+0.000608 i \\
W_{430}=0.00350-0.00515 i\end{array}$ \\
\hline
\end{tabular}

Note that only $W_{l m 0}$ are listed here, and that the other non-zero coeffs are linearly dependent on them.

the originals shown in Figure 5 from which the velocity profiles were determined, and the following conclusions are drawn from this comparison:

First, it is evident that for the first two textures, the contours in both the (001) and (111) pole figures of the recovered plots capture the hot spots of the original distributions well. The preferred orientations of (111) in the centre for Texture-1 and the three hot spots of the (001) distribution for Texture-2 are both clearly recognizable. For Texture-3, even though the contours of the recovered pole figures appear to be different from the original distributions, in fact the random texture is captured and the pole distribution densities should be near uniform for all poles which is well reflected in terms of the distribution of intensities. Therefore, the (001) and (111) pole figures recovered from wave velocities are demonstrated to provide good quality information about the original ODF.

It is observed that the smallest values in some of the reconstructed plots are negative. This results for two reasons: firstly, the simulated velocities used to determine the ODF coefficients inversely are different from theoretical values, as compared in Section 5.3, hence errors are introduced (because of grain interaction etc); and secondly, the truncation degree of 4 means the expansion is not a perfect approximation to the original function, so localized deviations may occur. Moreover, the truncation errors are also responsible for the greatly reduced intensities at the preferred orientations in the reconstructed pole figures for the first two original textures with strongly preferred orientations. According to Roe [8], the error can be quantitatively calculated via:

$$
\delta_{w}=\sum_{l=6}^{\infty} \sum_{m=-l}^{l} \sum_{n=-l}^{l} W_{l m n} W_{l m n}^{*}
$$




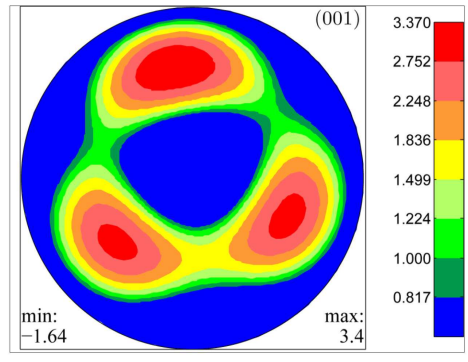

a

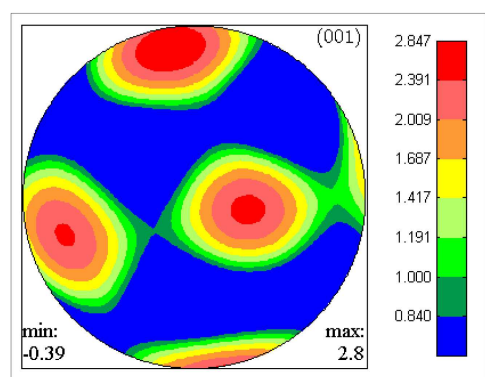

c

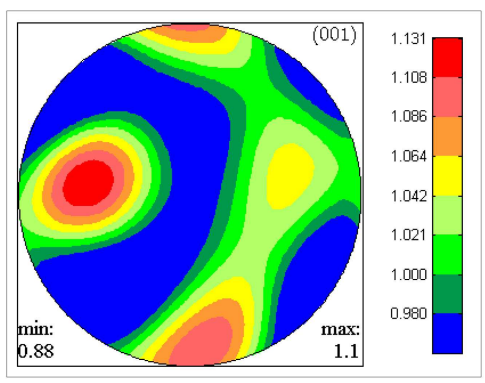

e

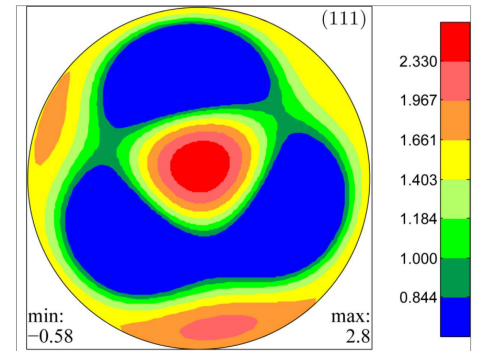

b

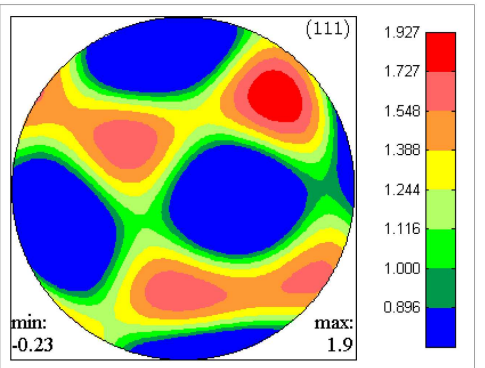

d

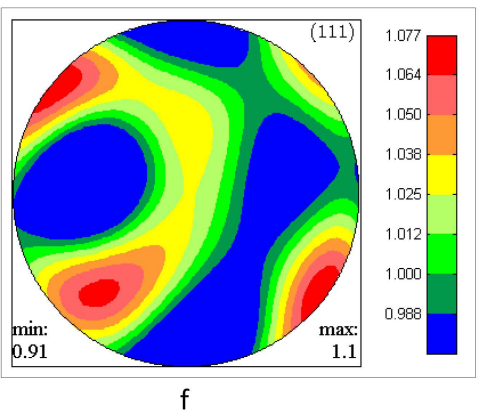

Figure 10: The pole figures of the three ODFs retrieved from wave velocities, where $\mathrm{a}$ and $\mathrm{b}$ are: the (001) and (111) pole figures of Texture-1, respectively; $c$ and d: plots of Texture-2; and e and f: plots of Texture-3.

and the error is likely to be quite large. However, even for the truncated ODF, random texture and single crystal orientations are still the two extreme cases which would set up the lower and upper bounds for the amplitudes, and it is possible that the values obtained with these extremes also truncated at 4th degree would enable us to re-scale the intensities of the recovered ODFs to comparable values to the originals.

\section{Conclusions}

The spherical harmonic convolution approach obtained specifically for HCP materials in [1] has been expanded into a generalised form which applies for all types of crystal structures. It has been shown that the ODF of a polycrystalline aggregate convolves with the single crystal wave velocity function to give the polycrystal velocity function.

When applying the generalised theorem for forward studies, the polycrystal velocity function for any crystal type can be predicted with knowledge of the ODF and the kernel. Representative studies have been performed on the cubic metal Fe, with both theoretical orientations and experimental textures, 
and satisfactory predictions of the polycrystal velocities are obtained.

When applying the generalised theorem for inverse studies, however, it is shown that only for HCP and cubic structure can the ODF be obtained from polycrystal velocities. In the studies on the Fe kernel convolving with experimental textures, the reason for the fact that it is often not possible to recover ODF coefficients with 6th degrees or higher through ultrasonic tests has also been demonstrated. Further, proof of principle of the inverse technique has been established using simulated velocities from representative experimental textures, and the 4th degree ODF coefficients have been well recovered.

\section{Appendix A Angular differences between phase velocity and particle displacement directions for HCP and cubic single crystals}

For the known elastic matrix $\mathbf{c}$ of a material and an arbitrarily given wavefront normal $\mathbf{n}$, deductions of the Christoffel equation finally conclude as

$$
\left[c_{i j k l} n_{j} n_{k}-\rho v^{2} \delta_{i} l\right]\left[p_{l}\right]=0,
$$

where the subscripts stand for the tensor component indices, $\rho$ is the material's density, $\delta$ is the Kronecker product and the vector $\mathbf{p}$ is the particle displacement vector (or referred to as the polarization vector). It now transpires that equation A.1 is a classical eigenvalue-eigenvector problem; its three eigenvalues are directly linked to the phase velocities, of which the largest one is the longitudinal or quasi-longitudinal wave velocity, depending on whether its corresponding eigenvector is exactly parallel with $\mathbf{n}$ or not. As both $\mathbf{n}$ and $\mathbf{p}$ are unit vectors, the angle between them is hence equal to $\arccos (\mathbf{n} \cdot \mathbf{p})$.
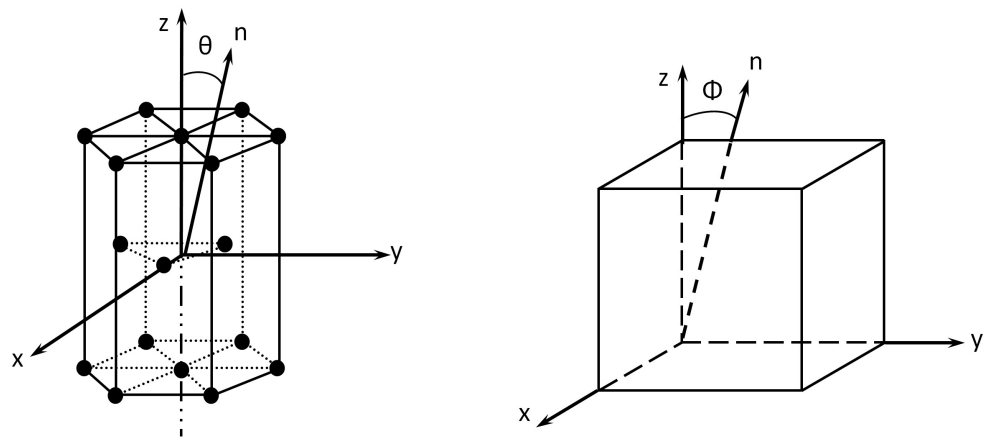

Figure A.1: The orientations of an HCP and a cubic crystal to be studied. The vector $\mathbf{n}$ varies on the x-z plane, with the angles $\theta \in[0, \pi / 2]$ and $\Phi \in[0, \pi / 4]$.

Our intention here is to evaluate the angular differences between the vectors $\mathbf{n}$ and $\mathbf{p}$ for $\mathrm{HCP}$ and cubic crystals; and as an example, only $\mathbf{n}$ varying on the x-z planes for the crystals with orientations shown in Figure A.1 are studied. It can be proved [29] that for the HCP crystal in Figure A.1a, $\theta \in[0, \pi / 2]$ is the smallest symmetric component region to evaluate the angular difference; while for the cubic crystal in Figure A.1b, $\Phi \in[0, \pi / 4]$ is the smallest region. $\alpha$-phased Ti-6Al-4V and Fe, whose elastic stiffness components are shown in Table A.1, are studied as the exemplary materials, and the angular differences for selections of $\theta$ and $\Phi$ are listed in Table A.2. It is thus clear that the largest difference between $\mathbf{n}$ and $\mathbf{p}$ for an HCP crystal is less than 3 degrees but escalate to more 
than 10 degrees for the cubic, so the former difference is often neglected while the latter needs to be taken account of for accurate calculations.

Table A.1: Single crystal elastic moduli $\alpha$ phase Ti-6Al-4V and Fe, for the former $C_{66}=\left(C_{11}-C_{12}\right) / 2$ (Units:GPa).

\begin{tabular}{cccccc}
\hline \hline & $C_{11}$ & $C_{12}$ & $C_{13}$ & $C_{33}$ & $C_{44}$ \\
\hline Ti-6Al-4V( $\alpha$ phase $)$ & 170.0 & 92.0 & 70.0 & 192.0 & 52.0 \\
Fe & 228.09 & 133.48 & - & - & 110.87 \\
\hline \hline
\end{tabular}

Table A.2: Angular differences between $\mathbf{n}$ and $\mathbf{p}$ for the HCP and cubic crystals at certain directions

\begin{tabular}{cc|cc}
\hline \multicolumn{2}{c|}{ HCP(Ti-6Al-4V) } & \multicolumn{2}{c}{ Cubic(Fe) } \\
\hline$\theta$ & $\begin{array}{c}\text { angle between } \mathbf{n} \\
\text { and p (deg) }\end{array}$ & $\Phi$ & $\begin{array}{c}\text { angle between } \mathbf{~} \\
\text { and p (deg) }\end{array}$ \\
\hline 0 & 0.00 & $\pi / 32$ & 0.00 \\
\hline$\pi / 16$ & 1.39 & $\pi / 16$ & 5.64 \\
\hline$\pi / 8$ & 2.42 & $3 \pi / 32$ & 10.29 \\
\hline $3 \pi / 16$ & 2.84 & $\pi / 8$ & 9.69 \\
\hline$\pi / 4$ & 2.58 & $5 \pi / 32$ & 7.99 \\
\hline $5 \pi / 16$ & 1.85 & $3 \pi / 16$ & 5.63 \\
\hline $3 \pi / 8$ & 1.04 & $7 \pi / 32$ & 2.90 \\
\hline $7 \pi / 16$ & 0.42 & $\pi / 4$ & 0.00 \\
\hline$\pi / 2$ & 0.00 &
\end{tabular}

\section{References}

[1] B. Lan, M. J.S. Lowe, and F. P. E. Dunne. A speherical harmonic approach to hcp texture from ultrasound: A solution to the inverse problem. Submitted to the Journal of the Mechanics and Physics of Solids.

[2] U. Fred Kocks, Carlos Norberto Tome, and H. R. Wenk. Texture and anisotropy: preferred orientations in polycrystals and their effect on materials properties. Cambridge university press, 2000.

[3] D. McKie and C. McKie. Essentials of crystallography. Crystalline solids. Blackwell Scientific, 1986.

[4] Jian-Min Zhang, Yan Zhang, Ke-Wei Xu, and Vincent Ji. Young's modulus surface and poisson's ratio curve for cubic metals. Journal of Physics and Chemistry of Solids, 68(4):503 - 510, 2007. 
[5] CA Bronkhorst, SR Kalidindi, and L Anand. Polycrystalline plasticity and the evolution of crystallographic texture in fcc metals. Philosophical Transactions of the Royal Society of London. Series A: Physical and Engineering Sciences, 341(1662):443-477, 1992.

[6] I.L Dillamore and W.T Roberts. Rolling textures in f.c.c. and b.c.c. metals. Acta Metallurgica, 12(3):281 - 293, 1964.

[7] J. Hirsch and K. Lucke. Overview no. 76: Mechanism of deformation and development of rolling textures in polycrystalline f.c.c. metals-i. description of rolling texture development in homogeneous cuzn alloys. Acta Metallurgica, 36(11):2863 - 2882, 1988.

[8] R. J. Roe. Description of crystallite orientation in polycrystalline materials .3. general solution to pole figure inversion. Journal of Applied Physics, 36(6):2024-2031, 1965.

[9] Bertram Eugene Warren. X-ray Diffraction. Courier Dover Publications, 1969.

[10] Adam J Schwartz, Mukul Kumar, Brent L Adams, and David P Field. Electron backscatter diffraction in materials science. Springer, 2009.

[11] Angus J. Wilkinson and T. Ben Britton. Strains, planes, and ebsd in materials science. Materials Today, 15(9):366-376, 2012.

[12] Jan Achenbach. Wave propagation in elastic solids. Elsevier, 1984.

[13] C. M. Sayers. Ultrasonic velocities in anisotropic polycrystalline aggregates. Journal of Physics D-Applied Physics, 15(11):2157-2167, 1982.

[14] R.B. Thompson, S.S. Lee, and J.F. Smith. Relative anisotropies of plane waves and guided modes in thin orthorhombic plates: Implication for texture characterization. Ultrasonics, 25(3):133137, 1987.

[15] P. J. Kielczynski, A. Moreau, and J. F. Bussiere. Determination of texture coefficients in hexagonal polycrystalline aggregates with orthorhombic symmetry using ultrasounds. Journal of the Acoustical Society of America, 95(2):813-827, 1994.

[16] S. Dixon, C. Edwards, and S. B. Palmer. Texture measurements of metal sheets using wideband electromagnetic acoustic transducers. Journal of Physics D-Applied Physics, 35(8):816-824, 2002. PII S0022-3727(02)30108-6.

[17] S. Matthies, G.W. Vinel, and K. Helming. Standard distributions in texture analysis: maps for the case of cubic-orthorhomic symmetry. Standard Distributions in Texture Analysis: Maps for the Case of Cubic-orthorhomic Symmetry. Akademie-Verlag, 1987.

[18] B. Lan, M. Lowe, and F. P. E. Dunne. Experimental and computational studies of ultrasound wave propagation in hexagonal close-packed polycrystals for texture detection. Acta Materialia, 63:107-122, 2014.

[19] R.E. Newnham. Properties of Materials : Anisotropy, Symmetry, Structure: Anisotropy, Symmetry, Structure. OUP Oxford, 2004.

[20] Jun Jiang, T. Benjamin Britton, and Angus J. Wilkinson. Mapping type \{III $\}$ intragranular residual stress distributions in deformed copper polycrystals. Acta Materialia, 61(15):5895 $5904,2013$. 
[21] M. A. Morrison and G. A. Parker. A guide to rotations in quantum mechanics. Australian Journal of Physics, 40:465, 1987.

[22] Morris Edgar Rose. Elementary theory of angular momentum. Courier Dover Publications, 1995.

[23] Peter J Kostelec and Daniel N Rockmore. Soft: So (3) fourier transforms. Department of Mathematics, Dartmouth College, Hanover, NH, 3755, 2007.

[24] A.D. Rollett. Orientation distribution:definition, discrete forms, examples. University Lecture, 2014.

[25] F Bachmann, Ralf Hielscher, and Helmut Schaeben. Texture analysis with mtex-free and open source software toolbox. Solid State Phenomena, 160:63-68, 2010.

[26] J. Roe, R. Inversion of pole figures for materials having cubic crystal symmetry. Journal of Applied Physics, 37:2069-2072, 1966.

[27] Martin J. Mohlenkamp. A user's guide to spherical harmonics, 2011.

[28] JAR Blais and Dean Allen Provins. Spherical harmonic analysis and synthesis for global multiresolution applications. Journal of Geodesy, 76(1):29-35, 2002.

[29] B.A. Auld. Acoustic fields and waves in solids. Number v. 2 in Acoustic Fields and Waves in Solids. R.E. Krieger, 1990. 